G. $\mathrm{Lu}$

Nagoya Math. J.

Vol. 181 (2006), 149-184

\title{
SYMPLECTIC CAPACITIES OF TORIC MANIFOLDS AND RELATED RESULTS
}

\author{
GUANGCUN LU*
}

\begin{abstract}
In this paper we give concrete estimations for the pseudo symplectic capacities of toric manifolds in combinatorial data. Some examples are given to show that our estimates can compute their pseudo symplectic capacities. As applications we also estimate the symplectic capacities of the polygon spaces. Other related results are impacts of symplectic blow-up on symplectic capacities, symplectic packings in symplectic toric manifolds, the Seshadri constant of an ample line bundle on toric manifolds, and symplectic capacities of symplectic manifolds with $S^{1}$-action.
\end{abstract}

\section{§1. Introduction and main results}

The symplectic capacities are the important tools of study of symplectic topology. There are several symplectic capacities. The typical two of them are the Gromov symplectic width $\mathcal{W}_{G}$ and Hofer-Zehnder capacity $c_{H Z}$ (cf. $[\mathrm{Gr}]$ and [HZ]). However, for a general symplectic manifold $(M, \omega)$ it is very difficult to compute or estimate $\mathcal{W}_{G}(M, \omega)$ and $c_{H Z}(M, \omega)$; see [Gin], [Lu3] and reference therein for the related results. It is well-known that the toric manifolds are a very beautiful family of Kähler manifolds admitting a combinatorial description. They also are rational and thus uniruled. So their pseudo symplectic capacities all are finite (cf. [Lu3]). The main aim of this paper is to estimate their (pseudo) symplectic capacities in terms of combinatorial data. Part results were announced in [Lu2] though they should be restricted to toric Fano manifolds as showed below.

Firstly, we briefly recall the typical pseudo symplectic capacity introduced in [Lu3]. For its properties and applications the reader refer to [Lu3]. Given a connected symplectic manifold $(M, \omega)$ of dimension $2 n$ and a smooth function $H$ on it let $X_{H}$ denote the symplectic gradient

Received December 15, 2004.

Revised June 11, 2005, August 9, 2005.

2000 Mathematics Subject Classification: 53D35, 37J45, 52B20.

*Partially supported by the NNSF 19971045 and 10371007 of China. 
of $H$. An isolated critical point $p$ of $H$ is called admissible if the spectrum of the linear transformation $D X_{H}(p): T_{p} M \rightarrow T_{p} M$ is contained in $\mathbb{C} \backslash\{\lambda i \mid 2 \pi \leq \pm \lambda<+\infty\}$. For two given nonzero homology classes $\alpha_{0}, \alpha_{\infty} \in H_{*}(M)$ we denote by

$$
\mathcal{H}_{a d}\left(M, \omega ; \alpha_{0}, \alpha_{\infty}\right) \quad\left(\operatorname{resp} . \quad \mathcal{H}_{a d}^{\circ}\left(M, \omega ; \alpha_{0}, \alpha_{\infty}\right)\right)
$$

the set of all smooth functions on $M$ for which there exist two smooth compact submanifolds $P$ and $Q$ of $M$ with connected smooth boundaries and of codimension zero such that the following condition groups $(a)(b)(c)(d)(e)(f)$ (resp. $\left.(\mathrm{a})(\mathrm{b})(\mathrm{c})(\mathrm{d})(\mathrm{e})\left(\mathrm{f}^{\prime}\right)\right)$ are satisfied:

(a) $P \subset \operatorname{Int}(Q)$ and $Q \subset \operatorname{Int}(M)$;

(b) $\left.H\right|_{P}=0$ and $\left.H\right|_{M-\operatorname{Int}(Q)}=\max H$;

(c) $0 \leq H \leq \max H$;

(d) There exist chain representatives of $\alpha_{0}$ and $\alpha_{\infty}$, still denoted by $\alpha_{0}$, $\alpha_{\infty}$, such that $\operatorname{supp}\left(\alpha_{0}\right) \subset \operatorname{Int}(P)$ and $\operatorname{supp}\left(\alpha_{\infty}\right) \subset M \backslash Q ;$

(e) There are no critical values in $(0, \varepsilon) \cup(\max H-\varepsilon$, $\max H)$ for a small $\varepsilon=\varepsilon(H)>0 ;$

(f) The Hamiltonian system $\dot{x}=X_{H}(x)$ on $M$ has no nonconstant periodic solutions of period less than 1 ;

$\left(\mathrm{f}^{\prime}\right)$ The Hamiltonian system $\dot{x}=X_{H}(x)$ on $M$ has no nonconstant contractible periodic solutions of period less than 1 .

If $\alpha_{0} \in H_{0}(M)$ can be represented by a point we allow $P$ to be an empty set. If $M$ is a closed manifold and $\alpha_{\infty} \in H_{0}(M)$ is represented by a point, we also allow $Q=M$.

The pseudo symplectic capacities of Hofer-Zehnder type are defined by

$$
\left\{\begin{array}{l}
C_{H Z}^{(2)}\left(M, \omega ; \alpha_{0}, \alpha_{\infty}\right):=\sup \left\{\max H \mid H \in \mathcal{H}_{a d}\left(M, \omega ; \alpha_{0}, \alpha_{\infty}\right)\right\} \\
C_{H Z}^{(2 \circ)}\left(M, \omega ; \alpha_{0}, \alpha_{\infty}\right):=\sup \left\{\max H \mid H \in \mathcal{H}_{a d}^{\circ}\left(M, \omega ; \alpha_{0}, \alpha_{\infty}\right)\right\} .
\end{array}\right.
$$

Denote by $p t$ the generator of $H_{0}(M)$ represented by a point. Then we have symplectic invariants

$$
\left\{\begin{array}{l}
C_{H Z}(M, \omega):=C_{H Z}(M, \omega ; p t, p t) \\
C_{H Z}^{\circ}(M, \omega):=\widehat{C}_{H Z}(M, \omega ; p t, p t)
\end{array}\right.
$$


It had been proved in [Lu3] that $C_{H Z}$ is a symplectic capacity and that $\mathcal{W}_{G} \leq C_{H Z} \leq c_{H Z}$. In fact, in Lemma 1.4 of the recent [Lu3, v9] we proved that $C_{H Z}(M, \omega)=c_{H Z}(M, \omega)$ and $C_{H Z}^{\circ}(M, \omega)=c_{H Z}^{\circ}(M, \omega)$ if either $M$ is closed or each compact subset $K \subset M \backslash \partial M$ may be contained in a compact submanifold $W \subset M$ with connected boundary and of codimension zero. Without special statements we follow all notations and conventions in [Lu3]. Especially, we always make the convention that $\sup \emptyset=0$ and $\inf \emptyset=+\infty$ in this paper. Moreover, we shall omit the superscripts in $C_{H Z}^{(2)}$ and $C_{H Z}^{(2 \circ)}$ without occurring of confusion.

A $2 n$-dimensional symplectic toric manifold is a closed connected symplectic manifold $(M, \omega)$ equipped with an effective Hamiltonian action $\tau$ : $\mathbb{T}^{n} \rightarrow \operatorname{Diff}(M, \omega)$ of the standard (real) $n$-torus $\mathbb{T}^{n}=\mathbb{R}^{n} / 2 \pi \mathbb{Z}^{n}$ and with a choice of a corresponding moment map $\mu: M \rightarrow\left(\mathbb{R}^{n}\right)^{*}$. The image $\triangle=\mu(M) \subset\left(\mathbb{R}^{n}\right)^{*}$ is a convex polytope, called the moment polytope. It was proved in [Del] that the polytope satisfies: (i) there are $n$ edges meeting at each vertex $p$, (ii) the edges meeting at the vertex $p$ are rational, i.e., each edge is of the form $p+t v_{k}, 0 \leq t \leq \infty$, where $v_{k} \in\left(\mathbb{Z}^{n}\right)^{*}$; (iii) the $v_{1}, \ldots, v_{n}$ in (ii) can be chosen to be a basis of $\left(\mathbb{Z}^{n}\right)^{*}$. Such a polytope is called a Delzant polytope. It can be uniquely written as

$$
\triangle=\bigcap_{k=1}^{d}\left\{x \in\left(\mathbb{R}^{n}\right)^{*} \mid\left\langle x, u_{k}\right\rangle=x\left(u_{k}\right) \geq \lambda_{k}\right\} .
$$

Here $d$ is the number of the $(n-1)$-dimensional faces of $\triangle, u_{k}$ is a uniquely primitive element of the lattice $\mathbb{Z}^{n} \subset \mathbb{R}^{n}$ (the inward-pointing normal to the $k$-th face of $\triangle$ ), and $\lambda_{k}$ is a real number. Delzant [Del] associated to this $\triangle \subset\left(\mathbb{R}^{n}\right)^{*}$ a closed connected symplectic manifold $\left(M_{\triangle}, \omega_{\triangle}\right)$ of dimension $2 n$ together with a Hamiltonian $\mathbb{T}^{n}$-action $\tau_{\triangle}: \mathbb{T}^{n} \rightarrow \operatorname{Diff}\left(M_{\triangle}, \omega_{\triangle}\right)$ such that the image of the corresponding moment map $\mu_{\triangle}: M_{\triangle} \rightarrow\left(\mathbb{R}^{n}\right)^{*}$ is precisely $\triangle$ and that $(M, \omega, \tau)$ is isomorphic as a Hamiltonian $\mathbb{T}^{n}$-space to $\left(M_{\triangle}, \omega_{\triangle}, \tau_{\triangle}\right)$. Two symplectic toric manifolds are isomorphic if they are equivariantly symplectomorphic. Two Delzant polytopes in $\left(\mathbb{R}^{n}\right)^{*}$ are isomorphic if they are differ by the composition of a translation with an element of $\operatorname{SL}(n, \mathbb{Z})$. Delzant showed in [Del] that two symplectic toric manifolds are isomorphic if and only if their Delzant polytopes are isomorphic. Thus we reduce the study of symplectic topology of $(M, \omega, \tau)$ to that of $\left(M_{\triangle}, \omega_{\triangle}, \tau_{\triangle}\right)$. Delzant's construction also yielded a "canonical" $\mathbb{T}^{n}$ invariant complex structure $J_{\triangle}$ compatible with the symplectic form $\omega_{\triangle}$. In other words the quadruple $\left(M_{\triangle}, \omega_{\triangle}, J_{\triangle}, \tau_{\triangle}\right)$ is a Kähler manifold. 
Such manifolds may be explained as a special class of projective varieties. There exists a class of normal algebraic varieties, called toric varieties, which are classified by combinatorial objects called fans. Let $\Sigma$ be a complete regular fan in $\mathbb{R}^{n}$ and $G(\Sigma)=\left\{u_{1}, \ldots, u_{d}\right\}$ be the set of all generators of 1-dimensional cones in $\Sigma$. Denote by $X_{\Sigma}$ the compact toric manifold associated with $\Sigma$. If it is projective, i.e., if $X_{\Sigma}$ admits a compatible symplectic structure $\omega$ such that $\left(X_{\Sigma}, \omega, J\right)$ is Kähler, then every Kähler form on $X_{\Sigma}$ can be represented by a strictly convex support function $\varphi$ for $\Sigma$ (cf. Section 2.2). Conversely every strictly convex support function for $\Sigma$ represents a Kähler form on $X_{\Sigma}$. Therefore, in this paper we shall use the same letter to denote a Kähler form on $X_{\Sigma}$ and the corresponding strictly convex support function for $\Sigma$ when the context makes our meaning clear. For such a function $\varphi$ setting

$$
\triangle_{\varphi}=\left\{x \in\left(\mathbb{R}^{n}\right)^{*} \mid\langle x, m\rangle \geq-\varphi(m) \forall m \in \mathbb{R}^{n}\right\}
$$

it is a Delzant polytope in $\left(\mathbb{R}^{n}\right)^{*}$. With $S^{1}=\{z \in \mathbb{C}|| z \mid=1\}$ the action of the maximal compact torus $\mathbb{T}^{n}=\mathbb{R}^{n} / 2 \pi \mathbb{Z}^{n} \cong\left(S^{1}\right)^{n} \in\left(\mathbb{C}^{*}\right)^{n}$ is Hamiltonian with respect to the symplectic structure $2 \pi \cdot \varphi$ and has moment polytope $\triangle_{\varphi}$. In other words

$$
\left(X_{\Sigma}, 2 \pi \cdot \varphi\right)=\left(M_{\triangle_{\varphi}}, \omega_{\triangle_{\varphi}}\right)
$$

For the Delzant polytope $\triangle$ in $(1.3)$ we denote by $\Sigma_{\triangle}$ the complete regular fan in $\mathbb{R}^{n}$ associated with it and by $P_{\triangle}=X_{\Sigma_{\triangle}}$ the corresponding projective toric manifold (cf. Section 2.2). It follows from (1.5) that $\Sigma$ is the fan associated with $\triangle_{\varphi}$. As showed in [Gu2] the set of all generators of 1-dimensional cones in $\Sigma_{\triangle}$ is given by $G\left(\Sigma_{\triangle}\right)=\left\{u_{1}, \ldots, u_{d}\right\}$, and under the identity $M_{\triangle}=P_{\triangle}$ the Kähler form $\omega_{\triangle}$ is represented by the strictly convex support function for $\Sigma_{\triangle}$ defined by $\omega_{\triangle}\left(u_{i}\right)=-2 \pi \lambda_{i}, i=1, \ldots, d$. Therefore $\triangle_{\omega_{\triangle}}=2 \pi \triangle$. So we can study the symplectic topology of toric manifolds from two points of view. Let $\mathbb{Z}_{\geq 0}$ be the set of all nonnegative integers. Denote by

$$
\Lambda(\Sigma, \varphi):=\max \sum_{i=1}^{d} \varphi\left(u_{i}\right) a_{i}
$$

where $\left(a_{1}, \ldots, a_{d}\right) \in \mathbb{Z}_{\geq 0}^{n}$ satisfies $\sum_{i=1}^{d} a_{i} u_{i}=0$ and $1 \leq \sum_{i=1}^{d} a_{i} \leq n+1$. Our first result is: 
THEOREM 1.1. For the above $\Sigma$ and $\varphi$ one has

$$
\begin{gathered}
0<\Lambda(\Sigma, \varphi) \leq(n+1) \max _{i} \varphi\left(u_{i}\right) \quad \text { and } \\
\mathcal{W}_{G}\left(X_{\Sigma}, \varphi\right) \leq C\left(X_{\Sigma}, \varphi ; p t, P D([\varphi])\right) \leq \Lambda(\Sigma, \varphi)
\end{gathered}
$$

for $C=C_{H Z}^{(2)}, C_{H Z}^{(2 \circ)}$ and $n \geq 2$. Moreover, for the interior $\operatorname{Int}\left(\triangle_{\varphi}\right)$ of $\triangle_{\varphi}$ it always holds that

$$
\mathcal{W}_{G}\left(X_{\Sigma}, \varphi\right) \geq \frac{1}{2 \pi} \mathcal{W}_{G}\left(\operatorname{Int}\left(\triangle_{\varphi}\right) \times \mathbb{T}^{n}, \omega_{\text {can }}\right)
$$

where $\left(\operatorname{Int}\left(\triangle_{\varphi}\right) \times \mathbb{T}^{n}, \omega_{\text {can }}\right)=\left(\left\{(x, \theta) \mid x \in \operatorname{Int}\left(\triangle_{\varphi}\right), \quad \theta \in \mathbb{R}^{n} / 2 \pi \mathbb{Z}^{n}\right\}\right.$, $\left.\sum_{k=1}^{d} d x_{k} \wedge d \theta_{k}\right)$.

As a by-product of proof of (1.8) we obtain in Corollary 3.2 Mori's theorem on the existence of rational curves through any point on a uniruled manifold with a different method. In Remark 1.5 below we shall give an example to show that $\Lambda(\Sigma, \varphi)$ may be much smaller than $(n+1) \max _{i} \varphi\left(u_{i}\right)$. In some condition the estimate in (1.8) can be improved.

TheOREM 1.2. If $X_{\Sigma}$ is also Fano, i.e., the anticanonical divisor $-K_{X_{\Sigma}}$ is ample, then

$\Upsilon(\Sigma, \varphi):=\inf \left\{\sum_{k=1}^{d} \varphi\left(u_{k}\right) a_{k}>0 \mid \sum_{k=1}^{d} a_{k} u_{k}=0, a_{k} \in \mathbb{Z}_{\geq 0}, k=1, \ldots, d\right\}>0$,

and for $C=C_{H Z}^{(2)}, C_{H Z}^{(2 \circ)}$ and any $n \geq 2$,

$$
\mathcal{W}_{G}\left(X_{\Sigma}, \varphi\right) \leq C\left(X_{\Sigma}, \varphi ; p t, P D([\varphi])\right) \leq \Upsilon(\Sigma, \varphi)
$$

By the definition it is easy to see that $\Upsilon(\Sigma, \varphi) \leq \Lambda(\Sigma, \varphi)$. In Theorem 2.3 we shall list three equivalent criterions to judge whether or not $X_{\Sigma}$ is Fano from $\Sigma$.

Let $\triangle^{n}(a):=\left\{\left(x_{1}, \ldots, x_{n}\right) \in \mathbb{R}_{>0}^{n} \mid \sum_{k=1}^{n} x_{k}<a\right\}$. For $\triangle$ in (1.3) the following number

$$
\mathcal{W}(\triangle):=\sup \left\{a>0 \mid \exists \Psi \in \operatorname{SL}(n, \mathbb{Z}), x \in\left(\mathbb{R}^{n}\right)^{*} \text { s.t. } \Psi\left(\triangle^{n}(a)\right)+x \subset \triangle\right\}
$$

is an invariant of the Delzant polytopes in $\left(\mathbb{R}^{n}\right)^{*}$ under the group generated by elements of $\operatorname{SL}(n, \mathbb{Z})$ and translations. For each vertex $p$ of $\triangle$ we can 
assign a positive number $E_{p}(\triangle)$ as follows. Let $p_{1}, \ldots, p_{n}$ be $n$ vertex adjacent to $p$. By the above definition of Delzant polytope we may assume that $p_{k}$ sits in an edge of the form $p+t v_{k}, t \geq 0, k=1, \ldots, n$. Denote by $r_{p}(\triangle)_{k}=\left|p-p_{k}\right| /\left|v_{k}\right|, k=1, \ldots, n$. Here $|v|$ denotes the standard norm of vector $v$ in $\left(\mathbb{R}^{n}\right)^{*}$. Then $p_{k}=p+r_{p}(\triangle)_{k} v_{k}, k=1, \ldots, n$. Let

$$
r_{p}(\triangle)=\left\{r_{p}(\triangle)_{1}, \ldots, r_{p}(\triangle)_{n}\right\} \quad \text { and } \quad E_{p}(\triangle)=\min _{1 \leq k \leq n} r_{p}(\triangle)_{k}
$$

Proposition 1.3. For the Delzant polytope $\triangle$ in (1.3) it holds that

$$
\frac{1}{2 \pi} \mathcal{W}_{G}\left(\operatorname{Int}(\triangle) \times \mathbb{T}^{n}, \omega_{\text {can }}\right) \geq \mathcal{W}(\triangle) \geq \max _{p \in \operatorname{Vert}(\triangle)} E_{p}(\triangle) .
$$

We also want to derive the estimation in terms of $\triangle$. A $n$-dimensional integral polytope $\triangle \subset\left(\mathbb{R}^{n}\right)^{*}$ was called reflexive in [Ba2] if it satisfies: (i) $\operatorname{Int}(\triangle) \cap\left(\mathbb{Z}^{n}\right)^{*}=\{0\}$, and (ii) all facets $F$ of $\triangle$ are supported by an affine hyperplane of the form $\left\{m \in\left(\mathbb{R}^{n}\right)^{*} \mid\left\langle m, v_{F}\right\rangle=-1\right\}$ for some $v_{F} \in \mathbb{Z}^{n}$. A equivalent version is that $0 \in \operatorname{Int}(\triangle)$ and the polar $\triangle^{0}:=\left\{x \in \mathbb{R}^{n} \mid\right.$ $\langle m, x\rangle \geq-1, \forall m \in \triangle\}$ is also a $n$-dimensional integral polytope $\mathbb{R}^{n}$. A reflexive polytope $\triangle$ is called a Fano polytope if the fan $\Sigma_{\triangle}$ is regular. Clearly, a reflexive and Delzant polytope is also Fano. Note that polytopes $\triangle$ and $r \cdot \triangle$ yield the same fans for any $r>0$, and that two toric manifolds corresponding with two isomorphic Delzant polytopes have same Fanoness. Thus a toric manifold $P_{\triangle}$ is Fano if and only if $r \cdot(m+\triangle)$ is a Fano polytope for some $m \in\left(\mathbb{R}^{n}\right)^{*}$ and $r>0$. In Theorem 2.5 we shall show that the toric manifold $P_{\triangle}$ associated with a Delzant polytope $\triangle$ in (1.3) is Fano if and only if there exist $m \in\left(\mathbb{R}^{n}\right)^{*}$ and $r>0$ such that

$$
\begin{gathered}
\operatorname{Int}(r \cdot(m+\triangle)) \cap\left(\mathbb{Z}^{n}\right)^{*}=\{0\} \quad \text { and } \\
r \cdot\left(\lambda_{i}+\left\langle m, u_{i}\right\rangle\right)= \pm 1, \quad \forall 1 \leq i \leq d .
\end{gathered}
$$

More sufficient and necessary conditions will be given there. Using this we get the following corollary of Theorems 1.1 and 1.2.

Corollary 1.4. For the Delzant polytope $\triangle \subset\left(\mathbb{R}^{n}\right)^{*}$ in (1.3) let $\Lambda(\triangle)\left(=\Lambda\left(\Sigma_{\triangle}, \omega_{\triangle}\right)\right)$ be the maximum of $-2 \pi \sum_{i=1}^{d} \lambda_{i} a_{i}$ for all $\left(a_{1}, \ldots, a_{d}\right)$ $\in \mathbb{Z}_{\geq 0}^{n}$ satisfying $\sum_{i=1}^{d} a_{i} u_{i}=0$ and $1 \leq \sum_{i=1}^{d} a_{i} \leq n+1$. Then $\Lambda(\triangle) \leq$ $-2 \pi(n+1) \min _{i} \lambda_{i}$ and for $C=C_{H Z}^{(2)}, C_{H Z}^{(2 \circ)}$ and $n \geq 2$,

$$
2 \pi \mathcal{W}(\triangle) \leq \mathcal{W}_{G}\left(M_{\triangle}, \omega_{\triangle}\right) \leq C\left(M_{\triangle}, \omega_{\triangle} ; p t, P D\left(\left[\omega_{\triangle}\right]\right)\right) \leq \Lambda(\triangle)
$$


If there exist $r>0$ and $m \in\left(\mathbb{R}^{n}\right)^{*}$ such that $r \cdot(m+\triangle)$ satisfies (1.15), then

$$
\Upsilon(\triangle):=\inf \left\{-\sum_{k=1}^{d} \lambda_{k} a_{k}>0 \mid \sum_{k=1}^{d} a_{k} u_{k}=0, a_{k} \in \mathbb{Z}_{\geq 0}, k=1, \ldots, d\right\}>0,
$$

and for $C=C_{H Z}^{(2)}, C_{H Z}^{(2 \circ)}$ and any $n \geq 2$ it holds that

$$
\mathcal{W}_{G}\left(M_{\triangle}, \omega_{\triangle}\right) \leq C\left(M_{\triangle}, \omega_{\triangle} ; p t, P D\left(\left[\omega_{\triangle}\right]\right)\right) \leq 2 \pi \Upsilon(\triangle) .
$$

Remark 1.5. The polygon space associated with $\alpha=\left(\alpha_{1}, \ldots, \alpha_{5}\right)=$ $(3 / 2,1,1,1,4 / 3)$ is a symplectic toric manifold $\left(\operatorname{Pol}(\alpha), \omega_{\alpha}\right)$ with moment polytope $\triangle_{\alpha}$ given by

$$
\begin{aligned}
\left\{\left(x_{1}, x_{2}\right) \in \mathbb{R}^{2} \mid\right. & \frac{1}{2} \leq x_{1} \leq \frac{5}{2}, \frac{1}{3} \leq x_{2} \leq \frac{7}{3} \\
& \left.x_{1}+x_{2} \geq 1, x_{1}-x_{2} \geq-1, x_{2}-x_{1} \geq-1\right\} .
\end{aligned}
$$

(cf. $[\mathrm{HaKn}]$ ). Using (1.15) one can prove that it is not Fano. We can also compute that $\Lambda\left(\triangle_{\alpha}\right)=25 \pi / 3<15 \pi=-2 \pi(n+1) \min _{i} \lambda_{i}$. This shows that the second inequality in (1.7) may be strict. Since $\Psi=\operatorname{diag}(1,-1) \in$ $\mathrm{SL}(2, \mathbb{Z})$ and $\Psi\left(\triangle^{2}(1)\right)+\left(\frac{1}{2}, \frac{3}{2}\right)$ is contained in $\triangle_{\alpha}$, we get that $\mathcal{W}\left(\triangle_{\alpha}\right) \geq 1$. From these we can use (1.16) to obtain

$$
2 \pi \leq \mathcal{W}_{G}\left(\operatorname{Pol}(\alpha), \omega_{\alpha}\right) \leq C\left(\operatorname{Pol}(\alpha), \omega_{\alpha} ; p t, P D\left(\left[\omega_{\alpha}\right]\right)\right) \leq 25 \pi / 3
$$

Moreover, we can prove that $\Upsilon\left(\triangle_{\alpha}\right)=1 / 6$. So the second inequality in (1.18), i.e.,

$$
C\left(\operatorname{Pol}(\alpha), \omega_{\alpha} ; p t, P D\left(\left[\omega_{\alpha}\right]\right)\right) \leq 2 \pi \Upsilon\left(\triangle_{\alpha}\right)=\pi / 3
$$

can not hold because the first one in (1.18) always hold. These show that the second inequalities in (1.11) and (1.18) do not necessarily hold for nonFano symplectic toric manifolds.

Notice that $\left(a_{1}, \ldots, a_{d}\right) \in \mathbb{Z}_{\geq 0}^{n}$ is only taken over a finite set in the definition of $\Lambda(\Sigma, \varphi)$. Using the formula in [Sp] it might be possible to get the optimal estimation for any compact non-Fano toric manifold.

The rest of the paper is organized as follows. In Section 2 we give some necessary preliminaries on toric manifolds; the readers only need to browse through them. The main results are proved in Section 3. Three examples are given in Section 4. In Section 5 we estimate symplectic capacities 
of the polygon spaces. Finally four related results are given in Section 6; They are impacts of symplectic blow-ups on symplectic capacities, symplectic packings in toric manifolds and the estimate of Seshadri constants of an ample line bundle on toric manifolds, and symplectic capacities of symplectic manifolds with $S^{1}$-action.

Acknowledgement. I am grateful to Professors V. V. Batyrev, A. Givental, A. Kresch, H. Sato, B. Siebert and J. A. Wiśniewski for clarifying some facts. The author also thanks ICTP at Italy and IHES at Paris for their financial support and hospitality.

\section{§2. Preliminaries on toric manifolds}

The basic references for toric manifolds (in alphabetic order) are $[\mathrm{Au}]$, [Ba1], [Ew], [Fu], [Gu2] and [Oda]. The description here will be presented in the unity notations in [Ba1] and [Gu2].

\subsection{Symplectic toric manifolds}

Let $\left(M_{\triangle}, \omega_{\triangle}, J_{\triangle}, \tau_{\triangle}\right)$ be the symplectic toric manifold associated with Delzant polytope $\triangle \subset\left(\mathbb{R}^{n}\right)^{*}$ in $(1.3)$, and $\mu_{\triangle}: M_{\triangle} \rightarrow\left(\mathbb{R}^{n}\right)^{*}$ be the moment map of the $\mathbb{T}^{n}$-action $\tau_{\triangle}$ on it. Denote by $F_{k}$ the $k$-th $(n-1)$-dimensional face of $\triangle$ defined by the equation $\left\langle x, u_{k}\right\rangle=\lambda_{k}$. They yield complex and symplectic submanifolds of $M_{\triangle}$ of real codimension 2 ,

$$
D_{1}=\mu_{\triangle}^{-1}\left(F_{1}\right), \ldots, D_{d}=\mu_{\triangle}^{-1}\left(F_{d}\right) .
$$

Let $c_{k}$ be the cohomology class in $H^{2}\left(M_{\triangle}, \mathbb{Z}\right)$ dual to $D_{k}$. The cohomology class $\left[\omega_{\triangle}\right]$ and the first Chern class of $M_{\triangle}$ are respectively given by

$$
\frac{1}{2 \pi}\left[\omega_{\triangle}\right]=-\sum_{k=1}^{d} \lambda_{k} c_{k} \quad \text { and } \quad c_{1}\left(M_{\triangle}\right)=\sum_{k=1}^{d} c_{k}
$$

(cf. [Gu1]). As pointed out in [Ab] the arguments in [Gu1] gave a symplectomorphism

$$
\left(\operatorname{Int}\left(M_{\triangle}\right), \omega_{\triangle}\right) \cong\left(\operatorname{Int}(\triangle) \times \mathbb{T}^{n}, \omega_{\text {can }}\right)
$$

Here $\operatorname{Int}\left(M_{\triangle}\right)=\phi_{\mathbb{T}^{n}}^{-1}(\operatorname{Int}(\triangle))$ is an open dense subset in $M_{\triangle}, x \in \operatorname{Int}(\triangle)$, $\theta \in \mathbb{R}^{n} / 2 \pi \mathbb{Z}^{n}$ and $\omega_{\text {can }}=\sum_{k=1}^{d} d x_{k} \wedge d \theta_{k}$. Thus $(x, \theta)$ may be viewed as symplectic coordinates in $\operatorname{Int}\left(M_{\triangle}\right)$. 


\subsection{Fans and toric varieties}

For an integer $k \geq 1$, a convex subset $\sigma \subset \mathbb{R}^{n}$ is called a regular $k$ dimensional cone if there exists a $\mathbb{Z}$-basis $v_{1}, \ldots, v_{k}, \ldots, v_{n}$ of $\mathbb{Z}^{n}$ such that $\sigma=\mathbb{R}_{\geq 0} v_{1}+\cdots+\mathbb{R}_{\geq 0} v_{k}$. Such $v_{1}, \ldots, v_{k} \in \mathbb{Z}^{n}$ are called the integral generators of $\sigma$. The origin $0 \in \mathbb{R}^{n}$ is called the regular zero dimensional cone. The cones generated by subsets of the integral generators of $\sigma$ are called the faces of $\sigma$. A finite system $\Sigma=\left\{\sigma_{1}, \ldots, \sigma_{s}\right\}$ of regular cones in $\mathbb{R}^{n}$ is called a complete regular $n$-dimensional fan in $\mathbb{R}^{n}$ if (i) any face of each cone $\sigma \in \Sigma$ is also in $\Sigma$; (ii) the intersection $\sigma_{1} \cap \sigma_{2}$ of any two cones $\sigma_{1}, \sigma_{2} \in \Sigma$ is a face of each; (iii) $\mathbb{R}^{n}=\sigma_{1} \cup \cdots \cup \sigma_{s}$. A toric variety is compact and nonsingular if and only if its corresponding fan is complete and regular. We always consider such a fan $\Sigma$ below. The set of all $k$-dimensional cones of $\Sigma$ is denoted by $\Sigma^{(k)}$. For every $\sigma \in \Sigma^{(1)}$ there is a unique generator $u \in \mathbb{Z}^{n}$ such that $\sigma=\mathbb{Z}_{\geq 0} \cdot u$. Denote by $G(\Sigma)=\left\{u_{1}, \ldots, u_{d}\right\}$ the set of all generators of elements of $\Sigma^{(1)}$. A nonempty subset $\mathcal{P}=\left\{u_{i_{1}}, \ldots, u_{i_{k}}\right\} \subset$ $G(\Sigma)$ is called a primitive collection if it is not the set of generators of a $k$-dimensional cone in $\Sigma$, while for each generator $u_{i_{l}} \in \mathcal{P}$ the elements of $\mathcal{P} \backslash\left\{u_{i_{l}}\right\}$ generate a $(k-1)$-dimensional cone in $\Sigma$. Since $\Sigma$ is complete there exists a unique cone $\sigma(\mathcal{P}) \in \Sigma$ whose relative interior contains $u_{i_{1}}+\cdots+u_{i_{k}}$. Let $G(\sigma(\mathcal{P}))=\left\{u_{j_{1}}, \ldots, u_{j_{m}}\right\}$. We get a linear relation

$$
u_{i_{1}}+\cdots+u_{i_{k}}=c_{j_{1}} u_{j_{1}}+\cdots+c_{j_{m}} u_{j_{m}}, \quad c_{j_{s}}>0, \quad c_{j_{s}} \in \mathbb{Z} .
$$

(we allow $m=0$ if $u_{i_{1}}+\cdots+u_{i_{k}}=0$.) It is called the primitive relation for $\mathcal{P}$. The integer $\operatorname{deg}(\mathcal{P}):=k-\left(c_{1}+\cdots+c_{m}\right)$ is called the degree of $\mathcal{P}$. Denote by $\operatorname{PC}(\Sigma)$ the set of primitive collections of $\Sigma$. Let $\mathrm{A}(\mathcal{P})=\{z \in$ $\mathbb{C}^{d} \mid z_{i}=0$ if $\left.u_{i} \in \mathcal{P}\right\}$ and $Z(\Sigma)=\bigcup_{\mathcal{P}} \mathrm{A}(\mathcal{P})$, where $\mathcal{P}$ takes over $\operatorname{PC}(\Sigma)$. Put $U(\Sigma)=\mathbb{C}^{d} \backslash Z(\Sigma)$ and

$$
R(\Sigma)=\left\{\mu=\left(\mu_{1}, \ldots, \mu_{d}\right) \in \mathbb{Z}^{d} \mid \mu_{1} u_{1}+\cdots+\mu_{d} u_{d}=0\right\} .
$$

Clearly, $R(\Sigma)$ is isomorphic to $\mathbb{Z}^{d-n}$. Let $\left(e_{1}, \ldots, e_{d}\right)$ be the standard basis of $\mathbb{R}^{d}$. Define a linear map $\beta: \mathbb{R}^{d} \rightarrow \mathbb{R}^{n}, e_{k} \mapsto u_{k}, k=1, \ldots, d$. It maps $\mathbb{Z}^{d}$ onto $\mathbb{Z}^{n}$. Note that the map $\beta$ can be naturally extended to a map $\beta_{\mathbb{C}}: \mathbb{C}^{d} \rightarrow \mathbb{C}^{n}$ that maps $2 \pi i \mathbb{Z}^{d}$ onto $2 \pi i \mathbb{Z}^{n}$. We still denote by $\beta_{\mathbb{C}}$ the induced map from $T_{\mathbb{C}}^{d}:=\mathbb{C}^{d} / 2 \pi i \mathbb{Z}^{d}$ to $T_{\mathbb{C}}^{n}:=\mathbb{C}^{n} / 2 \pi i \mathbb{Z}^{n}$. Let $N_{\mathbb{C}}(\Sigma)$ be the kernel of this map. Using the group isomorphism $\mathrm{E}_{d}: T_{\mathbb{C}}^{d} \rightarrow\left(\mathbb{C}^{*}\right)^{d}$ given by

$$
[w]=\left[\left(w_{1}, \ldots, w_{d}\right)\right] \longmapsto\left(e^{w_{1}}, \ldots, e^{w_{d}}\right),
$$


we get a subgroup of $\left(\mathbb{C}^{*}\right)^{d}, \mathrm{D}(\Sigma):=\mathrm{E}_{d}\left(N_{\mathbb{C}}(\Sigma)\right)$. Explicitly, it is isomorphic to $\left(\mathbb{C}^{*}\right)^{d-n}$ as the Lie group. Moreover, $\mathrm{D}(\Sigma)$ acts freely and properly on $U(\Sigma)$. Thus the quotient $X_{\Sigma}=U(\Sigma) / \mathrm{D}(\Sigma)$ is a simply connected compact complex manifold of dimension $n$, called the compact toric manifold associated with $\Sigma$. Denote by

$$
D_{k}(\Sigma)=\left\{\left[\left(z_{1}, \ldots, z_{d}\right)\right] \in U(\Sigma) / \mathrm{D}(\Sigma) \mid z_{k}=0\right\}, \quad k=1, \ldots, d .
$$

They are complex submanifolds of $X_{\Sigma}$ of codimension one and form a basis for the group $\mathrm{T}_{\mathrm{N}} \operatorname{Div}\left(X_{\Sigma}\right)$ of $T_{N}=\left(\mathbb{C}^{*}\right)^{n}$-invariant divisors.

A continuous function $\varphi: \mathbb{R}^{n} \rightarrow \mathbb{R}$ is called $\Sigma$-piecewise linear if it is a linear function on every cone of $\Sigma$. Such a function is uniquely determined by its values on elements $u_{k} \in G(\Sigma)$. We also call $\varphi \in \operatorname{PL}(\Sigma)$ integral if $\varphi\left(\mathbb{Z}^{n}\right) \subset \mathbb{Z}$. Denote by $\operatorname{PL}(\Sigma)$ the space of all $\Sigma$-piecewise linear functions on $\mathbb{R}^{n}$. For $\varphi \in \operatorname{PL}(\Sigma)$ and $\mu \in R(\Sigma) \otimes \mathbb{R}$ the degree of $\mu$ relative to $\varphi$ is defined by $\operatorname{deg}_{\varphi}(\mu)=\sum_{k=1}^{d} \mu_{k} \varphi\left(u_{k}\right)$

Theorem 2.1. For $A \in H_{2}\left(X_{\Sigma}, \mathbb{Z}\right)$ let $\mu_{k}(A)$ denote the intersection numbers $A \cdot D_{k}(\Sigma), k=1, \ldots, d$. Then $\mu(A)=\left(\mu(A)_{1}, \ldots, \mu(A)_{d}\right) \in R(\Sigma)$ and the map

$$
H_{2}\left(X_{\Sigma}, \mathbb{Z}\right) \longrightarrow R(\Sigma), \quad A \longmapsto \mu(A)
$$

is an isomorphism. Denote by $\Xi_{\Sigma}$ the inverse of the isomorphism and its natural extension $R(\Sigma) \otimes \mathbb{R} \rightarrow H_{2}\left(X_{\Sigma}, \mathbb{R}\right)$. Moreover, the homomorphism $\varphi \mapsto \sum_{k=1}^{d} \varphi\left(u_{k}\right) P D\left(D_{k}(\Sigma)\right)$ from $\mathrm{PL}(\Sigma)$ to $H^{2}\left(X_{\Sigma}, \mathbb{R}\right)$ also induces an isomorphism

$$
\Xi^{\Sigma}: \operatorname{PL}(\Sigma) / M_{\mathbb{R}} \longrightarrow H^{2}\left(X_{\Sigma}, \mathbb{R}\right) .
$$

In particular, under the isomorphism $\Xi^{\Sigma}$ the first Chern class $c_{1}\left(X_{\Sigma}\right)$ is represented by the class of $\varphi_{c_{1}} \in \operatorname{PL}(\Sigma)$ such that $\varphi_{c_{1}}\left(u_{1}\right)=\cdots=\varphi_{c_{1}}\left(u_{d}\right)=$ 1. Furthermore, the degree-mapping induces the nondegenerate pairing deg: $\mathrm{PL}(\Sigma) / M_{\mathbb{R}} \times R(\Sigma) \otimes \mathbb{R} \rightarrow \mathbb{R}$ which coincides with the canonical intersection pairing $H^{2}\left(X_{\Sigma}, \mathbb{R}\right) \times H_{2}\left(X_{\Sigma}, \mathbb{R}\right) \rightarrow \mathbb{R}$.

A nonzero homology class $A \in H_{2}\left(X_{\Sigma}, \mathbb{Z}\right)$ is called very effective in $[\mathrm{Kr}]$ if $A \cdot D \geq 0$ for every toric divisor $D$. Let $\operatorname{VNE}\left(X_{\Sigma}\right)$ denote the set of very effective curve classes on $X_{\Sigma}$. Then under the isomorphism (2.6) it is given by $\operatorname{VNE}\left(X_{\Sigma}\right)=\mathbb{Z}_{\geq 0}^{d} \cap(R(\Sigma) \backslash\{0\})$. 
For each cone $\sigma=\left\langle u_{i_{1}}, \ldots, u_{i_{n-1}}\right\rangle \in \Sigma^{(n-1)}$ let $\left\langle u_{i_{1}}, \ldots, u_{i_{n-1}}, u_{i_{n}}\right\rangle$ and $\left\langle u_{i_{1}}, \ldots, u_{i_{n-1}}, u_{i_{n+1}}\right\rangle$ are the $n$-dimensional cones in $\Sigma$ which contains $\sigma$ as a face. Then there are unique integers $b_{i} \in \mathbb{Z}, i=1, \ldots, n+1$ with $b_{n}=b_{n+1}=1$, such that $b_{1} u_{i_{1}}+\cdots+b_{n} u_{i_{n}}+b_{n+1} u_{i_{n+1}}=0$. We define $v(\sigma)=\left(v(\sigma)_{1}, \ldots, v(\sigma)_{d}\right) \in R(\Sigma)$ by $v(\sigma)_{r}=b_{t}$ for $r=i_{t}$ and $1 \leq t \leq n+1$, and by $v(\sigma)_{r}=0$ otherwise. Under the isomorphism (2.6) it corresponds to the class in $H_{2}\left(X_{\Sigma}, \mathbb{Z}\right)$ represented by the $T_{N}$-stable closed subvariety $V(\sigma) \cong \mathbb{C} P^{1}$. So the intersection number is

$$
\left(D_{l}(\Sigma) \cdot V(\sigma)\right)= \begin{cases}b_{t} & l=i_{t}(1 \leq t \leq n-1) \\ 0 & \text { otherwise }\end{cases}
$$

If $X_{\Sigma}$ is projective the effective cone is given by $\mathrm{NE}\left(X_{\Sigma}\right)=\sum_{\sigma \in \Sigma^{(n-1)}}$ $\mathbb{R}_{\geq 0} v(\sigma)$.

A $\Sigma$-piecewise linear function $\varphi \in \operatorname{PL}(\Sigma)$ is called strictly convex support function for $\Sigma$ if (i) it is upper convex, i.e., $\varphi(x)+\varphi(y) \geq \varphi(x+y)$ $\forall x, y \in \mathbb{R}^{n}$, and (ii) the restrictions of it to any two different $n$-dimensional cones $\sigma_{1}, \sigma_{2} \in \Sigma$, are two different linear functions. Denote by $\varphi_{l} \in \operatorname{PL}(\Sigma)$ the unique functions determined by $\varphi_{l}\left(u_{k}\right)=\delta_{k l}, k, l=1, \ldots, d$. It is easily checked that they are all upper convex. Moreover, under the isomorphism (2.7) the divisor $D_{l}(\Sigma) \in H^{2}\left(X_{\Sigma}, \mathbb{R}\right)$ corresponds to the class represented by $\varphi_{l}$. Denote by $K(\Sigma)$ the cone in $H^{2}\left(X_{\Sigma}, \mathbb{R}\right) \cong \mathrm{PL}(\Sigma) /\left(\mathbb{R}^{n}\right)^{*}$ consisting of the classes of all upper convex $\varphi \in \operatorname{PL}(\Sigma)$, and by $K^{\circ}(\Sigma)$ the interior of $K(\Sigma)$, i.e., the cone consisting of the classes of all strictly convex support functions $\varphi \in \operatorname{PL}(\Sigma)$. Then $K^{\circ}(\Sigma) \neq \emptyset$ if and only if $X_{\Sigma}$ is projective.

THEOREM 2.2. For a complete regular fan $\Sigma$ in $\mathbb{R}^{n}, \varphi \in \operatorname{PL}(\Sigma)$ is a strictly convex support function for it if and only if the following equivalent conditions hold.

(i) For any primitive collection $\mathcal{P}=\left\{u_{i_{1}}, \ldots, u_{i_{k}}\right\} \subset G(\Sigma)$ it holds that

$$
\varphi\left(u_{i_{1}}\right)+\cdots+\varphi\left(u_{i_{k}}\right)>\varphi\left(u_{i_{1}}+\cdots+u_{i_{k}}\right) .
$$

(ii) $\triangle_{\varphi}:=\left\{m \in\left(\mathbb{R}^{n}\right)^{*} \mid\langle m, x\rangle \geq-\varphi(x), \forall x \in \mathbb{R}^{n}\right\}$ is a Delzant polytope in $\left(\mathbb{R}^{n}\right)^{*}$. In this case, for each maximal cone $\sigma \in \Sigma$ let $\varphi_{\sigma} \in\left(\mathbb{R}^{n}\right)^{*}$ be the unique element such that $\left\langle\varphi_{\sigma}, x\right\rangle=-\left.\varphi\right|_{\sigma}(x) \forall x \in \sigma$, then different maximal cone give different $\varphi_{\sigma} \in\left(\mathbb{R}^{n}\right)^{*}$ and $\left\{\varphi_{\sigma} \mid \sigma \in \Sigma^{(n)}\right\}$ is exactly the set of vertexes of $\triangle_{\varphi}$. 
(iii) The divisor $\sum_{k=1}^{d} \varphi\left(u_{k}\right) D_{k}$ is ample, or equivalently

$$
\left(\left(\sum_{l=1}^{d} \varphi\left(u_{l}\right) D_{l}(\Sigma)\right) \cdot V(\sigma)\right)=\sum_{k=1}^{d} \varphi\left(u_{k}\right) v(\sigma)_{k}>0 \quad \text { for all } \sigma \in \Sigma^{(n-1)} \text {. }
$$

(i) is Theorem 4.6 in [Ba1]. The first claim in (ii) follows from Corollary 2.15 in [Oda], and the second is Lemma 2.12 in [Oda]. (iii) is Theorem 2.18 in [Oda].

With the above fan $\Sigma$ one can associate a polytope in $\mathbb{R}^{n}$

$$
\triangle_{\Sigma}:=\bigcup_{\left\langle u_{1}, \ldots, u_{k}\right\rangle \in \Sigma} \operatorname{conv}\left(0, u_{1}, \ldots, u_{k}\right)
$$

where $u_{i} \in G(\Sigma)$ and $\left\langle u_{1}, \ldots, u_{k}\right\rangle$ is the convex cone spanned on vectors $u_{1}, \ldots, u_{k}$.

THEOREM 2.3. The compact toric manifold $X_{\Sigma}$ is Fano if and only if the following equivalent conditions hold.

(i) $\quad \Sigma$-piecewise linear function $\varphi_{c_{1}}$ defined in Theorem 2.1 is strictly convex for $\Sigma$.

(ii) Every primitive collection $\mathcal{P}$ of $\Sigma$ has positive degree.

(iii) The polytope $\triangle_{\Sigma}$ is strictly convex in the sense that each face of it is of the form $\operatorname{conv}\left(u_{i_{1}}, \ldots, u_{i_{k}}\right)$ where $\left\langle u_{i_{1}}, \ldots, u_{i_{k}}\right\rangle \in \Sigma$.

(i) and (ii) come from [Ba1] and [Ba3] respectively. (iii) was obtained on page 268 in $[\mathrm{Wi}]$.

There are only finitely many toric Fano varieties of dimension $n$ up to isomorphism. Toric Fano manifolds have been classified in low dimensions: there exist exactly 5 different toric Del Pezzo surfaces, exactly 18 different toric Fano 3-folds and exactly 124 different toric Fano 4-folds (see [Ba2], [Ba3], [Oda] and references therein).

Since a compact nonsingular toric variety $X_{\Sigma}$ is projective (or Kähler) if and only if its fan $\Sigma$ comes from some Delzant polytope, we recall the construction of the fan $\Sigma_{\triangle}$ associated with the Delzant polytope $\triangle$ in (1.3). For each face $F$ of $\triangle$ of codimension $k$ there exists a unique multi-index $I_{F}$ of length $k, I_{F}=\left(i_{1}, \ldots, i_{k}\right), 1 \leq i_{1}<\cdots<i_{k} \leq d$, such that $F=$ $\left\{x \in\left(\mathbb{R}^{n}\right)^{*} \mid x\left(u_{i}\right)=\lambda_{i}, \forall i \in I_{F}\right\}$. One has a regular $k$-dimensional cone in $\mathbb{R}^{n}, \sigma_{F}=\left\{\sum t_{i} u_{i} \mid t_{i} \geq 0 \forall i \in I_{F}\right\}$ with generators $\left\{u_{i} \mid i \in I_{F}\right\}$. The 
origin $0 \in \mathbb{R}^{n}$ is called the regular 0 -dimensional cone. Then the set $\Sigma_{\triangle}:=$ $\left\{\sigma_{F} \mid F\right.$ is a face of $\left.\triangle\right\}$ is a complete regular $n$-dimensional fan in $\mathbb{R}^{n}$ with $G\left(\Sigma_{\triangle}\right)=\left\{u_{1}, \ldots, u_{d}\right\}$, and the corresponding toric manifold $P_{\triangle}:=X_{\Sigma_{\triangle}}$ is projective. Audin showed in $[\mathrm{Au}]$ that there exists a biholomorphism from $\left(M_{\triangle}, J_{\triangle}\right)$ to $P_{\triangle}=U\left(\Sigma_{\triangle}\right) / \mathrm{D}\left(\Sigma_{\triangle}\right)$ which maps $D_{k}$ in $(2.1)$ to $D_{k}\left(\Sigma_{\triangle}\right)$ in $(2.5), k=1, \ldots, d$. Later we shall not distinguish between $M_{\triangle}$ and $P_{\triangle}$ without special statements. By Theorem 2.2 (ii), if $X_{\Sigma}$ is projective then any $\varphi \in K^{\circ}(\Sigma)$ yields a Delzant polytope $\triangle_{\varphi}$. It is easily proved that the fan $\Sigma$ associated with $\triangle_{\varphi}$ is exactly $\Sigma$. Moreover, for any $m \in\left(\mathbb{R}^{n}\right)^{*}$ and $r>0$, the above construction implies that $\Sigma_{m+r} \Delta=\Sigma_{\triangle}$ and thus $P_{m+r} \triangle=P_{\triangle}$ because $m+r \triangle=\bigcap_{k=1}^{d}\left\{x \in\left(\mathbb{R}^{n}\right)^{*} \mid x\left(u_{k}\right) \geq m\left(u_{k}\right)+r \lambda_{k}\right\}$.

THEOREM 2.4. For the Delzant polytope $\triangle$ in (1.3) the following assertions hold:

(i) $K^{\circ}\left(\Sigma_{\triangle}\right) \neq \emptyset$, and the open cone $K^{\circ}\left(\Sigma_{\triangle}\right) \subset H^{2}\left(P_{\triangle}, \mathbb{R}\right)=H^{1,1}\left(P_{\triangle}, \mathbb{R}\right)$ consists of classes of Kähler $(1,1)$-forms on $P_{\triangle}$. The support function $h_{\triangle}: \mathbb{R}^{n} \rightarrow \mathbb{R}$ for $\triangle$ defined by

$$
h_{\triangle}(x)=-\inf \{\langle v, x\rangle \mid v \in \triangle\} \quad \forall x \in \mathbb{R}^{n},
$$

is strictly convex for $\Sigma_{\triangle}$, and $\omega_{\triangle}=2 \pi h_{\triangle}$.

(ii) $\triangle_{\omega_{\triangle}}=2 \pi \triangle$, and if $\varphi \in \operatorname{PL}\left(\Sigma_{\triangle}\right)$ is strictly convex for $\Sigma_{\triangle}$ then one has

$$
\begin{gathered}
\triangle_{\varphi}=\bigcap_{i=1}^{d}\left\{m \in\left(\mathbb{R}^{n}\right)^{*} \mid\left\langle m, u_{i}\right\rangle \geq-\varphi\left(u_{i}\right)\right\} \quad \text { and } \\
\left(M_{\triangle_{\varphi}}, \omega_{\triangle_{\varphi}}\right)=\left(P_{\triangle}, 2 \pi \varphi\right) .
\end{gathered}
$$

(i) follows from Theorem 2.7 in [Oda]. To prove (ii), it is showed before that each $(n-1)$-dimensional face $F_{i}=\left\{m \in \triangle \mid\left\langle m, u_{i}\right\rangle=\lambda_{i}\right\}$ gives a corresponding 1-dimensional cone $\sigma_{F_{i}}=\mathbb{R}_{\geq 0} u_{i}$ in $\Sigma_{\triangle}$. By Lemma 2.12 in [Oda] this cone yields a $(n-1)$-dimensional face $F_{i}^{\varphi}:=\left\{m \in \triangle_{\varphi} \mid\left\langle m, u_{i}\right\rangle=\right.$ $\left.-\varphi\left(u_{i}\right)\right\}$ again.

THEOREM 2.5. The projective toric manifold $M_{\triangle}=P_{\triangle}$ is Fano if and only if the following equivalent conditions hold.

(i) There exist $r>0$ and $m \in\left(\mathbb{R}^{n}\right)^{*}$ such that $\operatorname{Int}(r \cdot(m+\triangle)) \cap\left(\mathbb{Z}^{n}\right)^{*}=$ $\{0\}$ and that $r \cdot\left(\lambda_{i}+\left\langle m, u_{i}\right\rangle\right)= \pm 1$ for $i=1, \ldots, d$. 
(ii) There exist $r>0$ and $m \in \mathbb{R}^{n}$ such that $0 \in \operatorname{Int}(m+\triangle)$ and that each vertex of $r \cdot(m+\triangle)$ is a primitive vector in $\left(\mathbb{Z}^{n}\right)^{*}$ in the sense that its coordinates are relatively prime.

Proof. To prove (i), note that $P_{\triangle}=P_{\mu(m+\triangle)}$. By Exercise 3.6 on page 70 of [Gu2], the vertices of the polytope $r \cdot(m+\triangle)$ lie on integer lattice points if and only if all $r \cdot\left(\lambda_{i}+\left\langle m, u_{i}\right\rangle\right)$ are integers, $i=1, \ldots, d$. Moreover it was proved in $[\mathrm{Ba} 2]$ that $P_{r \cdot(m+\triangle)}$ is Fano if and only if the integral polytope $r \cdot(m+\triangle)$ is a reflexive polytope. These imply (i). As to (ii) it was proved in $[\mathrm{Ew}]$ that $P_{r \cdot(m+\triangle)}$ is Fano if and only if the integral polytope $r \cdot(m+\triangle)$ is a Fano ploytope. The condition in (ii) just right guarantees that $r \cdot(m+\triangle)$ is a Fano polytope.

\section{§3. Proof of the Main Theorems}

Let $(M, \omega)$ be a closed symplectic manifold. For nonzero classes $\alpha_{0}, \alpha_{\infty} \in H_{*}(M, \mathbb{Q})$, using the Gromov-Witten invariant homomorphism $\Psi_{A, g, m+2}: H_{*}\left(\overline{\mathcal{M}}_{g, m+2} ; \mathbb{Q}\right) \times H_{*}(M ; \mathbb{Q})^{m+2} \rightarrow \mathbb{Q}$, we defined in [Lu3] a number $\mathrm{GW}_{g}\left(M, \omega ; \alpha_{0}, \alpha_{\infty}\right)$ by the infimum of the $\omega$-areas $\omega(A)$ of the homology classes $A \in H_{2}(M ; \mathbb{Z})$ for which $\Psi_{A, g, m+2}\left(\kappa ; \alpha_{0}, \alpha_{\infty}, \beta_{1}, \ldots, \beta_{m}\right) \neq 0$ for some homology classes $\beta_{1}, \ldots, \beta_{m} \in H_{*}(M ; \mathbb{Q}), \kappa \in H_{*}\left(\overline{\mathcal{M}}_{g, m+2} ; \mathbb{Q}\right)$ and integer $m>0$. It was proved in Theorem 1.10 and Remark 1.11 of [Lu3] that for $C=C_{H Z}^{(2)}, C_{H Z}^{(2 \circ)}$,

$$
\begin{gathered}
C\left(M, \omega ; \alpha_{0}, \alpha_{\infty}\right) \leq \mathrm{GW}_{0}\left(M, \omega ; \alpha_{0}, \alpha_{\infty}\right) \quad \text { and } \\
\mathrm{GW}_{g}(M, \omega ; p t, P D([\omega]))=\inf \left\{\mathrm{GW}_{g}(M, \omega ; p t, \alpha) \mid \alpha \in H_{*}(M, \mathbb{Q})\right\} .
\end{gathered}
$$

These are the starting points of proof of our main results.

\subsection{Rational curves on uniruled manifolds}

A smooth projective variety $X$ over $\mathbb{C}$ is called uniruled if it satisfies the following equivalent conditions:

(i) There is a nonempty open subset $U \subset X$ such that for every $x \in U$ there is a morphism $f: \mathbb{C} P^{1} \rightarrow X$ satisfying $x \in f\left(\mathbb{C} P^{1}\right)$.

(ii) For every $x \in X$ there is a morphism $f: \mathbb{C} P^{1} \rightarrow X$ satisfying $x \in$ $f\left(\mathbb{C} P^{1}\right)$.

The following proposition is a key to prove Theorem 1.1. Its proof was actually contained in Kollar's arguments in [Ko] and Proposition 7.3 in [Lu3]. For convenience of the readers we shall prove it in detail. 
Proposition 3.1. Let $X$ be a uniruled manifold of positive dimension $n$. Then there exist homology classes $A \in H_{2}(X ; \mathbb{Z})$ with $1 \leq c_{1}(A) \leq n+1$, $\alpha \in H_{2 n-2}(X, \mathbb{Q})$ and $\beta \in H_{*}(X ; \mathbb{Q})$ such that

$$
\Psi_{A, 0,3}(p t ; p t, \alpha, \beta) \neq 0 .
$$

Proof. Firstly, note that (3.3) and dimension condition in the definition of GW-invariants imply

$$
2+2 n+(2 n-\operatorname{dim} \beta)=2 n+2 c_{1}(A) .
$$

It follows that $1 \leq c_{1}(A) \leq n+1$ because $0 \leq \operatorname{dim} \beta \leq 2 n$. So we only need to prove (3.3).

Our proof ideas are based on the proof of Theorem 4.2.10 in [Ko] and simple arguments of Gromov-Witten invariants. Recall the proof of Theorem 4.2.10 in [Ko]. Fix a very general point $x \in X$ and a very ample divisor $H \subset X$. Since $X$ is uniruled there exists a rational curve $C$ through $x$ such that $(C \cdot H)$ is minimal. Let $B=:[C]$. Fix a point $z_{0} \in \mathbb{C} P^{1}$ and let $k$ be the complex dimension of the space of morphisms $f: \mathbb{C} P^{1} \rightarrow X$ such that $f_{*}\left(\left[\mathbb{C} P^{1}\right]\right)=B$ and $f\left(z_{0}\right)=x$. Then $k \geq 2$ because the isotropic subgroup of automorphism group of $\mathbb{C} P^{1}$ at $z_{0}$ has real dimension 4 . Then for general divisors $H_{1}, \ldots, H_{k}$ linearly equivalent to $H$,

$$
\Psi_{B, 0, k+1}\left(p t ; p t, H_{1}, \ldots, H_{k}\right) \neq 0 .
$$

If $k=2$ then (3.3) holds for $A=B$. If $k=3$ it follows from (6) in [Mc] that

$$
\begin{aligned}
& \Psi_{B, 0,4}\left(p t ; p t, H_{1}, H_{2}, H_{3}\right) \\
& \quad=\sum_{B=B_{1}+B_{2}} \sum_{l} \Psi_{B_{1}, 0,3}\left(p t ; p t, H_{1}, e_{l}\right) \Psi_{B_{2}, 0,3}\left(p t ; f_{l}, H_{2}, H_{2}\right)
\end{aligned}
$$

where $\left\{e_{l}\right\}_{l}$ is a basis for the homology $H_{*}(X ; \mathbb{Q})$ and $\left\{f_{l}\right\}_{l}$ is the dual basis with respect to the intersection pairing. This identity implies that $\Psi_{B_{1}, 0,3}\left(p t ; p t, H_{1}, e_{l}\right) \neq 0$ for some $l$. Taking $A=B_{1}$ one gets (3.3) again. If $k \geq 4$ the composition law of the $\mathrm{GW}$-invariants gives

$$
\begin{aligned}
& \Psi_{B, 0, k+1}\left(p t ; H_{1}, \ldots, H_{k}\right) \\
& =\sum_{B=B_{1}+B_{2}} \sum_{a, b} \Psi_{B_{1}, 0,4}\left(p t ; p t, H_{1}, H_{2}, \beta_{a}\right) \eta^{a b} \Psi_{B_{2}, 0, k-1}\left(p t ; \beta_{b}, H_{3}, \ldots, H_{k}\right) .
\end{aligned}
$$


Here $\left\{\beta_{b}\right\}_{b=1}^{L}$ is a homogeneous basis of $H_{*}(X, \mathbb{Q})$. It follows from (3.4) that

$$
\Psi_{B_{1}, 0,4}\left(p t ; p t, H_{1}, H_{2}, \beta_{a}\right) \neq 0
$$

for some $B_{1} \in H_{2}(X ; \mathbb{Z})$ and $1 \leq a \leq L$. As in case $k=3$ we can also get (3.3). Clearly we has always $\left(H^{\prime} \cdot A\right) \leq\left(H^{\prime} \cdot B\right)$ for any very ample divisor $H^{\prime}$ on $X$.

Corollary 3.2. For a uniruled manifold $X$ of positive dimension $n$, through any general point of $X$ there is a rational curve $C$ with $0<\left(-K_{X}\right.$. $C) \leq n+1$.

This result is not new. It is an easy part of the celebrated Mori's theorem in [Mor1], [Mor2]. For more general versions of Corollary 3.2 the reader may refer to [KoMor].

\subsection{Proof of Theorem 1.1}

Since $X_{\Sigma}$ is uniruled, Proposition 3.1 yields homology classes $A \in$ $H_{2}\left(X_{\Sigma} ; \mathbb{Z}\right)$ with $1 \leq c_{1}(A) \leq n+1, \alpha \in H_{2 n-2}\left(X_{\Sigma}, \mathbb{Q}\right)$ and $\beta \in H_{*}\left(X_{\Sigma} ; \mathbb{Q}\right)$ such that $\Psi_{A, 0,3}(p t ; p t, \alpha, \beta) \neq 0$. Note that the Gromov-Witten invariants are deformation invariants. For any $\varphi \in K^{\circ}(\Sigma)$ one has

$$
\langle[\varphi], A\rangle=\sum_{i=1}^{d} \varphi\left(u_{i}\right) \mu(A)_{i}>0 .
$$

Now $K(\Sigma)$ is the closure of $K^{\circ}(\Sigma)$ in $H^{2}\left(X_{\Sigma}, \mathbb{R}\right)$. Therefore $\langle[\psi], A\rangle=$ $\sum_{i=1}^{d} \psi\left(u_{i}\right) \mu(A)_{i} \geq 0$ for any $\psi \in K(\Sigma)$. In particular we have

$$
\left\langle\left[\varphi_{l}\right], A\right\rangle=\sum_{i=1}^{d} \varphi_{l}\left(u_{i}\right) \mu(A)_{i}=\mu(A)_{l} \geq 0, \quad l=1, \ldots, d .
$$

These show that $A$ is very effective. By Theorem 2.1, $c_{1}(A)=\sum_{i=1}^{d} \mu(A)_{i}$. So $1 \leq \sum_{i=1}^{d} \mu(A)_{i} \leq n+1$. By the definition of $\Lambda(\Sigma, \varphi)$ we have

$$
0<\langle[\varphi], A\rangle=\sum_{i=1}^{d} \varphi\left(u_{i}\right) \mu(A)_{i} \leq \Lambda(\Sigma, \varphi)
$$

and thus $\mathrm{GW}_{0}(M, \omega ; p t, \alpha) \leq \Lambda(\Sigma, \varphi)$. Moreover it is clear that

$$
\sum_{i=1}^{d} \varphi\left(u_{i}\right) \mu_{i} \leq \sum_{\varphi\left(u_{i}\right)>0} \varphi\left(u_{i}\right) \mu_{i} \leq(n+1) \max _{i} \varphi\left(u_{i}\right)
$$


for each $\mu \in \mathbb{Z}_{\geq 0}^{n}$ satisfying $\sum_{i=1}^{d} \mu_{i} u_{i}=0$ and $1 \leq \sum_{i=1}^{d} \mu_{i} \leq n+1$. By (3.1) and (3.2) we get the desired (1.8).

The proof of (1.9) is direct. Note that $\triangle_{\varphi}$ may be written as

$$
\triangle_{\varphi}=\bigcap_{k=1}^{d}\left\{x \in\left(\mathbb{R}^{n}\right)^{*} \mid\left\langle x, u_{k}\right\rangle=x\left(u_{k}\right) \geq-\varphi\left(u_{k}\right)\right\}
$$

By Theorem 2.4 (ii) it is a Delzant polytope in $\left(\mathbb{R}^{n}\right)^{*}$, and $\left(M_{\triangle_{\varphi}}, \omega_{\triangle_{\varphi}}\right)=$ $\left(P_{\triangle_{\varphi}}, 2 \pi \varphi\right)=\left(X_{\Sigma}, 2 \pi \varphi\right)$. Using $(2.3)$ we can give a symplectic embedding from $\left(\operatorname{Int}\left(\triangle_{\varphi}\right) \times \mathbb{T}^{n}, \omega_{\text {can }}\right)$ to $\left(M_{\triangle_{\varphi}}, \omega_{\triangle_{\varphi}}\right)$. Then (1.9) follows from these and the monotonicity of symplectic capacities.

\subsection{Proof of Theorem $\mathbf{1 . 2}$}

For every $A=\Xi_{\Sigma}(a) \in \operatorname{VNE}\left(X_{\Sigma}\right)$, by Theorem 9.1 in [Ba1] the moduli space $\mathcal{M}\left(A, X_{\Sigma}\right)$ consisting of holomorphic maps $f: \mathbb{C} P^{1} \rightarrow X_{\Sigma}$ with $f_{*}\left(\left[\mathbb{C} P^{1}\right]\right)=A$ is irreducible and the virtual dimension of it is equal to $n+c_{1}\left(X_{\Sigma}\right)(A)=n+\sum_{k=1}^{d} a_{k}$. Denote by $m=1+\sum_{k=1}^{d} a_{k}$ and by $c_{k} \in H^{2}\left(X_{\Sigma}, \mathbb{Z}\right)$ the Poincare dual of $\left[D_{k}(\Sigma)\right], k=1, \ldots, d$. It was stated in [Ba1] that

$$
c_{1}^{a_{1}} \cdots c_{d}^{a_{d}}=q^{A}
$$

holds in $Q H^{*}\left(X_{\Sigma}\right)$. The author incorrectly admitted it in [Lu2]. Actually one only can prove (3.6) for all $A \in \operatorname{VNE}\left(X_{\Sigma}\right)$ in the toric Fano manifolds. The first proof was given by Givental in [Giv] (also see $[\mathrm{Kr}]$ for an elementary proof for a class of Fano toric manifolds, and [CiS] for another different proof for Fano toric manifolds with minimal Chern number at least two). In terms of GW-invariants (3.6) means

$$
\Psi_{A, 0, m+1}^{X_{\Sigma}}(p t ; p t, \underbrace{D_{1}(\Sigma), \ldots, D_{1}(\Sigma)}_{a_{1}}, \ldots, \underbrace{D_{d}(\Sigma), \ldots, D_{d}(\Sigma)}_{a_{d}})=1
$$

Its enumerative interpretation is that for a given general point $p_{0}$ on $X_{\Sigma}$ and generic distinct points $z_{0}, z_{k, i}, i=1, \ldots, a_{k}$ and $k=1, \ldots, d$ on $\mathbb{C} P^{1}$ there exists precisely one morphism $f \in \mathcal{M}\left(A, X_{\Sigma}\right)$ such that $f\left(z_{0}\right)=p_{0}$ and $f\left(z_{k, i}\right) \in D_{k}(\Sigma)$ for $i=1, \ldots, a_{k}$ and $k=1, \ldots, d$. In particular $\varphi(A)>0$. But Theorem 2.1 shows that $\varphi(A)=\sum_{k=1}^{d} \omega\left(u_{k}\right) a_{k}$. Therefore for a given $a \in \mathbb{Z}_{\geq 0}^{d} \cap R(\Sigma), \Xi_{\Sigma}(a) \in \operatorname{VNE}\left(X_{\Sigma}\right)$ if and only if $\sum_{k=1}^{d} \varphi\left(u_{k}\right) a_{k}>0$. Now 
(1.10) can easily follow from this and the Gromov compactness theorem. Hence (3.2) and (3.7) give

$$
\mathrm{GW}_{0}\left(X_{\Sigma}, \varphi ; p t, P D([\varphi])\right) \leq \varphi(A)=\sum_{k=1}^{d} \varphi\left(u_{k}\right) a_{k}
$$

for any $A=\Xi_{\Sigma_{\triangle}}(a) \in \operatorname{VNE}\left(X_{\Sigma}\right)$, and thus

$$
\mathrm{GW}_{0}\left(X_{\Sigma}, \varphi ; p t, P D([\varphi])\right) \leq \Upsilon(\Sigma, \varphi)
$$

This and (3.1) give (1.11).

Remark 3.3. For a symplectic toric manifold $\left(\operatorname{Pol}(\alpha), \omega_{\alpha}\right)$ in Remark 1.5 it is easily seen that (3.6) cannot hold for all $A \in \operatorname{VNE}(\operatorname{Pol}(\alpha))$.

\subsection{Proof of Proposition 1.3}

Denote by $\omega_{0}=\sum_{k=1}^{n} d x_{k} \wedge d \theta_{k}$ and $\omega_{\text {can }}=\sum_{k=1}^{n} d x_{k} \wedge d \theta_{k}$ the standard symplectic form on $\mathbb{R}^{2 n}=\mathbb{R}^{n} \times \mathbb{R}^{n}$ and its descending symplectic form on $\mathbb{R}^{n} \times \mathbb{T}^{n}=\mathbb{R}^{n} \times\left(\mathbb{R}^{n} / 2 \pi \mathbb{Z}^{n}\right)$ respectively. For $a_{k}>0, b_{k}>0, k=1, \ldots, n$, we also denote by

$$
\begin{aligned}
& E\left(r_{1}, \ldots, r_{n}\right)=\left\{\left(x_{1}, y_{1}, \ldots, x_{n}, y_{n}\right) \in \mathbb{R}^{2 n} \mid \sum_{j=1}^{n}\left(x_{j}^{2}+y_{j}^{2}\right) / r_{j}^{2}<1\right\}, \\
& \triangle\left(a_{1}, \ldots, a_{n}\right)=\left\{\left(x_{1}, \ldots, x_{n}\right) \in \mathbb{R}_{>0}^{n} \mid \sum_{k=1}^{n} x_{k} / a_{k}<1\right\} \subset \mathbb{R}^{n}, \\
& \square\left(b_{1}, \ldots, b_{n}\right)=\left\{\left(\theta_{1}, \ldots, \theta_{n}\right) \in \mathbb{R}^{n} \mid 0<\theta_{k}<b_{k} \forall 1 \leq k \leq n\right\}
\end{aligned}
$$

and abbreviate $\triangle^{n}(a):=\triangle\left(a_{1}, \ldots, a_{n}\right)$ and $\square^{n}(b):=\square\left(b_{1}, \ldots, b_{n}\right)$ if $a_{1}=$ $\cdots=a_{n}=a$ and $b_{1}=\cdots=b_{n}=b$. The following two lemmas will be also used in Section 6.

LEMmA 3.4. ([Sik]) Let $U, V \subset \mathbb{R}^{n}$ be two connected open sets with $H^{1}(U)=0$ and $H^{1}(V)=0$. For the symplectic submanifolds $U \times \mathbb{T}^{n}$ and $V \times \mathbb{T}^{n}$ of $\left(\mathbb{R}^{n} \times \mathbb{T}^{n}, \omega_{\text {can }}\right)$, the following two statements are equivalent:

(i) $\left(U \times \mathbb{T}^{n}, \omega_{\text {can }}\right)$ and $\left(V \times \mathbb{T}^{n}, \omega_{\text {can }}\right)$ are symplectomorphic;

(ii) there exists a unimodular matrix $\Phi \in \mathbb{Z}^{n \times n}$ and a vector $x \in \mathbb{R}^{n}$ such that $V=\Phi U+x$. 
Lemma 3.5. ([Sch, Lemma 3.11]) Let $E\left(c_{1}, \ldots, c_{n}\right)$ be as above. Then for all $\epsilon>0$,

(i) $\left(E\left(\sqrt{2 a_{1}}-\epsilon, \ldots, \sqrt{2 a_{n}}-\epsilon\right), \omega_{0}\right)$ embeds symplectically in $\left(\triangle\left(a_{1}, \ldots\right.\right.$, $\left.\left.a_{n}\right) \times \square^{n}(2 \pi), \omega_{0}\right)$ in such a way that for all $\alpha \in(0,1), \alpha E\left(\sqrt{2 a_{1}}-\right.$ $\left.\epsilon, \ldots, \sqrt{2 a_{n}}-\epsilon\right)$ is mapped into $\left((\alpha+\epsilon) \triangle\left(a_{1}, \ldots, a_{n}\right)\right) \times \square^{n}(2 \pi)$;

(ii) $\left(\triangle\left(a_{1}-\epsilon, \ldots, a_{n}-\epsilon\right) \times \square^{n}(2 \pi), \omega_{0}\right)$ embeds symplectically in $E\left(\sqrt{2 a_{1}}\right.$, $\left.\ldots, \sqrt{2 a_{n}}\right)$ in such a way that for all $\alpha \in(0,1),\left(\alpha \triangle\left(a_{1}-\epsilon, \ldots, a_{n}-\right.\right.$ $\epsilon)) \times \square^{n}(2 \pi)$ is mapped into $(\alpha+\epsilon) E\left(\sqrt{2 a_{1}}, \ldots, \sqrt{2 a_{n}}\right)$.

Now we are in position to prove Proposition 1.3. By Lemma 3.4, if $\Psi\left(\triangle^{n}(a)\right)+x \subset \triangle$ for some $\Psi \in \operatorname{SL}(n, \mathbb{Z})$ and $x \in\left(\mathbb{R}^{n}\right)^{*}$ then there exists a symplectic embedding from $\left(\triangle^{n}(a) \times \mathbb{T}^{n}, \omega_{\text {can }}\right)$ into $\left(\triangle \times \mathbb{T}^{n}, \omega_{\text {can }}\right)$. Moreover, Lemma 3.5 can give a symplectic embedding from $\left(B^{2 n}(\sqrt{2 a}-\right.$ $\left.\epsilon), \omega_{0}\right)$ into $\left(\triangle^{n}(a) \times \square^{n}(2 \pi), \omega_{0}\right) \subset\left(\triangle^{n}(a) \times \mathbb{T}^{n}, \omega_{\text {can }}\right)$ for any given small $\epsilon>0$. The definition of $\mathcal{W}(\triangle)$ and the monotonicity of the symplectic capacities yield the first inequality in (1.14).

In order to prove the second inequality in (1.14) let $p \in \operatorname{Vert}(\triangle)$ such that

$$
E_{p}(\triangle)=\max \left\{E_{q}(\triangle) \mid q \in \operatorname{Vert}(\triangle)\right\}
$$

Suppose that $p_{1}, \ldots, p_{n} \in \operatorname{Vert}(\triangle)$ are the adjacent $n$ vertexes as described above Proposition 1.3. Then there exists a unique unimodular matrix $A \in$ $\operatorname{SL}(n, \mathbb{Z})$ such that $A e_{k}^{*}=v_{k}, k=1, \ldots, n$. So the map

$$
\Phi:\left(\mathbb{R}^{n}\right)^{*} \longrightarrow\left(\mathbb{R}^{n}\right)^{*}, \quad x \longmapsto A x-p
$$

maps the vertexes $p$ and $p_{1}, \ldots, p_{n}$ to the origin and $r_{p}(\triangle)_{1} e_{1}^{*}, \ldots, r_{p}(\triangle)_{n} e_{n}^{*}$. It follows that $\Phi$ maps the convex combination $\operatorname{conv}\left(p, p_{1}, \ldots, p_{n}\right)$ onto $\operatorname{conv}\left(0, r_{p}(\triangle)_{1} e_{1}^{*}, \ldots, r_{p}(\triangle)_{n} e_{n}^{*}\right)$. Since $\operatorname{conv}\left(p, p_{1}, \ldots, p_{n}\right) \subset \triangle$, the inverse map $\Phi^{-1}$ of $\Phi$ maps $\operatorname{conv}\left(0, r_{p}(\triangle)_{1} e_{1}^{*}, \ldots, r_{p}(\triangle)_{n} e_{n}^{*}\right)$ into $\triangle$. But $\triangle^{n}\left(E_{p}(\triangle)\right)$ is contained in $\operatorname{conv}\left(0, r_{p}(\triangle)_{1} e_{1}^{*}, \ldots, r_{p}(\triangle)_{n} e_{n}^{*}\right)$. The second inequality in (1.14) is obtained immediately.

\section{§4. Examples}

Example 4.1. In [CdFKM] Candelas, de la Ossa, Font, Katz, and Morrison resolved the curve of $\mathbb{Z}_{2}$ singularities of the weighted projective space $\mathbb{C} P^{4}(1,1,2,2,2)$ to obtain a compact toric manifold $X=X_{\Sigma}$. Here 
the one-dimensional cones in the fan $\Sigma$ are spanned by

$$
\begin{gathered}
u_{1}=-e_{1}-2 e_{2}-2 e_{3}-2 e_{4}, \quad u_{2}=e_{1}, \quad u_{3}=e_{2}, \\
u_{4}=e_{3}, \quad u_{5}=e_{4}, \quad u_{6}=\frac{1}{2}\left(u_{1}+u_{2}\right)
\end{gathered}
$$

for the standard basis $e_{1}, e_{2}, e_{3}, e_{4}$ in $\mathbb{C}^{4}$. The maximal cones of $\Sigma$ are as follows:

$$
\begin{array}{ll}
\sigma_{1}=\left\langle u_{1}, u_{3}, u_{4}, u_{5}\right\rangle, & \sigma_{5}=\left\langle u_{2}, u_{3}, u_{4}, u_{5}\right\rangle \\
\sigma_{2}=\left\langle u_{1}, u_{4}, u_{5}, u_{6}\right\rangle, & \sigma_{6}=\left\langle u_{2}, u_{4}, u_{5}, u_{6}\right\rangle \\
\sigma_{3}=\left\langle u_{1}, u_{3}, u_{5}, u_{6}\right\rangle, & \sigma_{7}=\left\langle u_{2}, u_{3}, u_{5}, u_{6}\right\rangle \\
\sigma_{4}=\left\langle u_{1}, u_{3}, u_{4}, u_{6}\right\rangle, & \sigma_{8}=\left\langle u_{2}, u_{3}, u_{4}, u_{6}\right\rangle .
\end{array}
$$

The only two primitive collections are $\left\{u_{1}, u_{2}\right\}$ and $\left\{u_{3}, u_{4}, u_{5}, u_{6}\right\}$. By Theorem 2.2 a $\Sigma$-piecewise linear function $\varphi \in \operatorname{PL}(\Sigma)$ is a strictly convex support function for $\Sigma$ if and only if $\varphi\left(u_{3}\right)+\varphi\left(u_{4}\right)+\varphi\left(u_{5}\right)+\varphi\left(u_{6}\right)>0$ and $\varphi\left(u_{1}\right)+\varphi\left(u_{2}\right)>2 \varphi\left(u_{6}\right)$. Note that $u_{1}+u_{2}=2 u_{6} \cdot \varphi_{c_{1}} \in \operatorname{PL}(\Sigma)$ is not a strictly convex support function for $\Sigma$. By Theorem 2.3, $X$ is not Fano. Let $\omega$ be the unique $\Sigma$-piecewise linear function determined by $\omega\left(u_{1}\right)=1$, $\omega\left(u_{3}\right)=1$ and $\omega\left(u_{i}\right)=0$ for $i=2,4,5,6$. It is easily checked that it is a strictly convex support function for $\Sigma$. So $X$ is projective and $\omega$ is a Kähler symplectic form. Theorem 1.1 yields

$$
\mathcal{W}_{G}(X, \omega) \leq C(X, \omega ; p t, P D([\omega])) \leq \Lambda(\Sigma, \omega)=1
$$

for $C=C_{H Z}^{(2)}, C_{H Z}^{(2 \circ)}$. Moreover, by Theorem 2.2 (ii) we can calculate all vertexes of $\triangle_{\omega}$ as follows:

$$
\begin{array}{lll}
t_{1}=(-1,-1,0,0), & t_{2}=(1,0,0,0), & t_{3}=(3,1,1,0), \\
t_{4}=(1,-1,0,1), & t_{5}=(0,-1,0,0), & t_{6}=(0,0,0,0), \\
t_{7}=(0,-1,1,0), & t_{8}=(0,-1,0,1) .
\end{array}
$$

Since the matrix

$$
\Phi=\left(\begin{array}{l}
t_{2}-t_{6} \\
t_{5}-t_{6} \\
t_{7}-t_{6} \\
t_{8}-t_{6}
\end{array}\right)=\left(\begin{array}{cccc}
1 & 0 & 0 & 0 \\
0 & -1 & 0 & 0 \\
0 & -1 & 1 & 0 \\
0 & -1 & 0 & 1
\end{array}\right)
$$


belongs to $\mathrm{SL}(4, \mathbb{Z})$ and maps $\mathrm{Cl}\left(\triangle^{n}(1)\right)=\operatorname{conv}\left\{0, e_{1}, e_{2}, e_{3}, e_{4}\right\}$ onto

$$
\operatorname{conv}\left\{t_{6}, t_{2}, t_{5}, t_{7}, t_{8}\right\} \subset \triangle_{\omega}
$$

It follows from Theorem 1.1 and Proposition 1.3 that

$$
\mathcal{W}_{G}(X, \omega) \geq \frac{1}{2 \pi} \mathcal{W}_{G}\left(\triangle_{\omega} \times \mathbb{T}^{n}, \omega_{\text {can }}\right) \geq \mathcal{W}\left(\triangle_{\omega}\right) \geq 1
$$

Combing (4.1) we get

$$
\mathcal{W}_{G}(X, \omega)=\frac{1}{2 \pi} \mathcal{W}_{G}\left(\triangle_{\omega} \times \mathbb{T}^{n}, \omega_{\text {can }}\right)=\mathcal{W}\left(\triangle_{\omega}\right)=1
$$

EXAMPLE 4.2. Let $\left(\mathbb{C} P^{n}, \omega_{\mathrm{FS}}\right)$ be $n$-dimensional projective space equipped with the Fubini-Study $\omega_{\mathrm{FS}}$. We assume that $\int_{\mathbb{C} P^{1}} \omega_{\mathrm{FS}}=2 \pi$. Then $\left(\mathbb{C} P^{n}, \omega_{\mathrm{FS}}\right)$ is a $2 n$-dimensional toric manifold and its Delzant polytope has vertices $q_{0}=0$ and $q_{i}=e_{i}, i=1, \ldots, n$. Here $e_{1}, \ldots, e_{n}$ are the standard basis of $\mathbb{R}^{n}$ and we have identified $\left(\mathbb{R}^{n}\right)^{*}$ with $\mathbb{R}^{n}$. Let $p \in \mathbb{C} P^{n}$ be a fixed point of action of $T^{n}$ on it corresponding vertex $q_{n}$ under the moment map. Since $\mathbb{C} P^{n}$ is Fano it easily follows from Corollary 1.4 that for $C=C_{H Z}^{(2)}, C_{H Z}^{(2 \circ)}$,

$$
\mathcal{W}_{G}\left(\mathbb{C} P^{n}, \omega_{\mathrm{FS}}\right)=C\left(\mathbb{C} P^{n}, \omega_{\mathrm{FS}} ; p t, P D\left(\left[\omega_{\mathrm{FS}}\right]\right)\right)=2 \pi .
$$

Now take $\tau \in(0,1)$ and consider the $\tau$-blow up of $\left(\mathbb{C} P^{n}, \omega_{\mathrm{FS}}\right)$ at $p$ we get a symplectic toric manifold $\left(\widetilde{\mathbb{C} P}_{\tau}^{n}, \omega_{\tau}\right)$. By Theorem 1.12 in [Gu2] the vertices of its Delzant polytope $\triangle_{\tau}$ are $q_{0}=0, q_{n}=\delta e_{n}$, and $q_{i}=e_{i}$, $q_{n+i}=\delta e_{n}+\delta e_{i}, i=1, \ldots, n-1$. Here $\delta=1-\tau$. It is easy to see that

$$
\triangle_{\tau}=\bigcap_{k=1}^{n+2}\left\{x \in \mathbb{R}^{n} \mid\left(x, u_{k}\right)-\lambda_{k} \geq 0\right\}
$$

where $u_{i}=e_{i}$ and $\lambda_{i}=0, i=1, \ldots, n$, and $u_{n+1}=-\sum_{i=1}^{m} e_{i}, u_{n+2}=-e_{n}$, $\lambda_{n+1}=-1$ and $\lambda_{n+2}=-\delta$. Note that all Delzant polytopes $\triangle_{\tau}$ generate the same fan. All toric manifolds $\widetilde{\mathbb{C P}}_{\tau}^{n}$ are same as complex manifolds. If $\tau=1 / 2$ it is easily checked that $2\left(\triangle_{1 / 2}-\left(\frac{1}{2}, \ldots, \frac{1}{2}\right)\right)$ satisfies $(1.15)$. So $\widetilde{\mathbb{C P}}_{1 / 2}^{n}$ is Fano. In particular we get that $(3.7)$ holds for $X_{\Sigma}=\widetilde{\mathbb{C} P}_{1 / 2}^{n}$. Note that the Kähler forms on $\widetilde{\mathbb{C} P}_{1 / 2}^{n}$ and $\widetilde{\mathbb{C} P}_{\tau}^{n}$ are deformedly equivalent because they sit in a Kähler cone on a complex manifold. Using the fact that the Gromov-Witten invariants are symplectic deformation invariants 
we still obtain $(3.7)$ for $X_{\Sigma}=\widetilde{\mathbb{C} P}_{\tau}^{n}$. As in the proof of Theorem 1.2 it follows from this and (3.1) that

$$
C\left(\widetilde{\mathbb{C P}}^{n}, \omega_{\tau} ; p t, P D\left(\left[\omega_{\tau}\right]\right)\right) \leq 2 \pi \Upsilon\left(\triangle_{\tau}\right)=2 \pi \delta
$$

for $C=C_{H Z}^{(2)}, C_{H Z}^{(2 \circ)}$. On the other hand the first inequality in (1.16) leads to

$$
\mathcal{W}_{G}\left(\widetilde{\mathbb{C} P}^{n}, \omega_{\tau}\right) \geq 2 \pi(1-\tau)
$$

because $\triangle^{n}(1-\tau) \subset \triangle$. Hence

$$
\mathcal{W}_{G}\left(\widetilde{\mathbb{C} P}^{n}, \omega_{\tau}\right)=C\left(\widetilde{\mathbb{C} P}^{n}, \omega_{\tau} ; p t, P D\left(\left[\omega_{\tau}\right]\right)\right)=2 \pi(1-\tau)
$$

for $C=C_{H Z}^{(2)}, C_{H Z}^{(2 \circ)}$. On the other hand it is easily checked that $\Lambda(\triangle) \geq$ $\pi(n+1)(1-\tau)$. So (1.18) gives better upper bound than (1.16) for $C_{H Z}\left(\widetilde{\mathbb{C P}}^{n}, \omega_{\tau} ; p t, P D\left(\left[\omega_{\tau}\right]\right)\right)$.

ExAmple 4.3. Consider the following 4-dimensional toric Fano manifold $W$ due to Hiroshi Sato [Sa, Ex. 4.7], which was missed in the table of Batyrev [Ba3]. Let $e_{1}, e_{2}, e_{3}, e_{4}$ be the standard basis in $\mathbb{R}^{4}$. Denote by $u_{1}=e_{1}, u_{2}=e_{2}, u_{3}=-e_{1}-e_{2}$ and $u_{4}=e_{3}, u_{5}=e_{4}, u_{6}=-e_{3}-e_{4}$. Let $W$ be the equivariant blow-ups of $\mathbb{C} P^{2} \times \mathbb{C} P^{2}$ along three $T_{N}$-invariant 2dimensional irreducible closed subvarieties $\overline{\operatorname{orb}\left(\left\{u_{1}, u_{4}\right\}\right)}, \overline{\operatorname{orb}\left(\left\{u_{2}, u_{5}\right\}\right)}$ and $\overline{\operatorname{orb}\left(\left\{u_{3}, u_{6}\right\}\right)}$. The set of all generators of 1-dimensional cones in its fan $\Sigma$ is $G(\Sigma)=\left\{u_{1}, u_{2}, u_{3}, u_{4}, u_{5}, u_{6}, u_{7}, u_{8}, u_{9}\right\}$, where $u_{7}=u_{1}+u_{4}, u_{8}=u_{2}+u_{5}$ and $u_{9}=u_{3}+u_{6}$. $\Sigma$ has 23 maximal cones as follows:

$$
\begin{array}{lll}
\sigma_{1}=\left\langle u_{1}, u_{2}, u_{7}, u_{8}\right\rangle, & \sigma_{2}=\left\langle u_{1}, u_{2}, u_{6}, u_{8}\right\rangle, & \sigma_{3}=\left\langle u_{1}, u_{2}, u_{6}, u_{7}\right\rangle, \\
\sigma_{4}=\left\langle u_{1}, u_{3}, u_{5}, u_{7}\right\rangle, & \sigma_{5}=\left\langle u_{1}, u_{3}, u_{5}, u_{9}\right\rangle, & \sigma_{6}=\left\langle u_{1}, u_{3}, u_{7}, u_{9}\right\rangle, \\
\sigma_{7}=\left\langle u_{1}, u_{5}, u_{6}, u_{8}\right\rangle, & \sigma_{8}=\left\langle u_{1}, u_{5}, u_{6}, u_{9}\right\rangle, & \sigma_{9}=\left\langle u_{1}, u_{5}, u_{7}, u_{8}\right\rangle, \\
\sigma_{10}=\left\langle u_{1}, u_{6}, u_{7}, u_{9}\right\rangle, & \sigma_{11}=\left\langle u_{2}, u_{3}, u_{4}, u_{9}\right\rangle, & \sigma_{12}=\left\langle u_{2}, u_{3}, u_{8}, u_{9}\right\rangle, \\
\sigma_{13}=\left\langle u_{2}, u_{3}, u_{4}, u_{5}\right\rangle, & \sigma_{14}=\left\langle u_{2}, u_{4}, u_{7}, u_{8}\right\rangle, & \sigma_{15}=\left\langle u_{2}, u_{4}, u_{6}, u_{7}\right\rangle, \\
\sigma_{16}=\left\langle u_{2}, u_{4}, u_{6}, u_{9}\right\rangle, & \sigma_{17}=\left\langle u_{2}, u_{6}, u_{8}, u_{9}\right\rangle, & \sigma_{18}=\left\langle u_{3}, u_{4}, u_{5}, u_{7}\right\rangle, \\
\sigma_{19}=\left\langle u_{3}, u_{4}, u_{7}, u_{9}\right\rangle, & \sigma_{20}=\left\langle u_{3}, u_{5}, u_{8}, u_{9}\right\rangle, & \sigma_{21}=\left\langle u_{4}, u_{5}, u_{7}, u_{8}\right\rangle, \\
\sigma_{22}=\left\langle u_{4}, u_{6}, u_{7}, u_{9}\right\rangle, & \sigma_{23}=\left\langle u_{5}, u_{6}, u_{8}, u_{9}\right\rangle . &
\end{array}
$$

Since $W$ is Fano, $\varphi_{c_{1}} \in \operatorname{PL}(\Sigma)$ defined by $\varphi_{c_{1}}\left(u_{i}\right)=1, i=1, \ldots, 9$ gives a symplectic structure on $W$. It is easily checked that

$$
\Upsilon\left(\Sigma, \varphi_{c_{1}}\right)=\inf \left\{\sum_{i=1}^{9} n_{i}>0 \mid \sum_{i=1}^{9} n_{i} u_{i}=0,\left(n_{1}, \ldots, n_{9}\right) \in\left(\mathbb{Z}_{\geq 0}\right)^{9} \backslash\{0\}\right\}=1 .
$$


By Theorem 1.2 we get that for $C=C_{H Z}^{(2)}, C_{H Z}^{(2 \circ)}$,

$$
\mathcal{W}_{G}\left(W, \varphi_{c_{1}}\right) \leq C\left(W, \varphi_{c_{1}} ; p t, P D\left(\left[\varphi_{c_{1}}\right]\right)\right) \leq 1
$$

By Theorem 2.2 (ii) we can calculate all vertexes of $\triangle_{\varphi_{c_{1}}}$ as follows:

$$
\begin{array}{lll}
t_{1}=(1,1,0,0), & t_{2}=(1,1,-1,0), & t_{3}=(1,1,0,-1), \\
t_{4}=(1,-2,0,1), & t_{5}=(1,-2,-1,1), & t_{6}=(1,-2,0,0), \\
t_{7}=(1,0,-2,1), & t_{8}=(1,-1,-2,1), & t_{9}=(1,0,0,1), \\
t_{10}=(1,-1,0,-1), & t_{11}=(-2,1,1,-1), & t_{12}=(0,-1,-2,-2), \\
t_{13}=(-2,1,1,1), & t_{14}=(0,1,1,0), & t_{15}=(0,1,1,-2), \\
t_{16}=(-2,-1,0,-1), & t_{17}=(-1,1,-1,0), & t_{18}=(0,-1,1,1), \\
t_{19}=(0,-1,-1,-1), & t_{20}=(-1,0,-1,1), & t_{21}=(0,0,1,1), \\
t_{22}=(0,0,1,-2), & t_{23}=(0,0,-2,1) . &
\end{array}
$$

Note that the matrix

$$
\Phi=\left(\begin{array}{c}
t_{2}-t_{1} \\
t_{3}-t_{1} \\
t_{9}-t_{1} \\
t_{14}-t_{1}
\end{array}\right)=\left(\begin{array}{cccc}
0 & 0 & -1 & 0 \\
0 & 0 & 0 & -1 \\
0 & -1 & 0 & -1 \\
-1 & 0 & 1 & 0
\end{array}\right)
$$

belongs to $\mathrm{SL}(4, \mathbb{Z})$ and maps $\mathrm{Cl}\left(\triangle^{n}(1)\right)=\operatorname{conv}\left\{0, e_{1}, e_{2}, e_{3}, e_{4}\right\}$ onto

$$
\operatorname{conv}\left\{t_{1}, t_{2}, t_{3}, t_{9}, t_{14}\right\}-t_{1} \subset \triangle_{\varphi_{c_{1}}}-t_{1} .
$$

It follows from Theorem 1.1 and Proposition 1.3 that

$$
\mathcal{W}_{G}\left(W, \varphi_{c_{1}}\right) \geq \frac{1}{2 \pi} \mathcal{W}_{G}\left(\triangle_{\varphi_{c_{1}}} \times \mathbb{T}^{n}, \omega_{\text {can }}\right) \geq \mathcal{W}\left(\triangle_{\varphi_{c_{1}}}\right) \geq 1
$$

Combing (4.3) we arrive at

$$
\mathcal{W}_{G}\left(W, \varphi_{c_{1}}\right)=C\left(W, \varphi_{c_{1}} ; p t, P D\left(\left[\varphi_{c_{1}}\right]\right)\right)=1
$$

for $C=C_{H Z}^{(2)}, C_{H Z}^{(2 \circ)}$.

\section{$\S 5$. Symplectic capacities of polygon spaces}

Let $\alpha=\left(\alpha_{1}, \ldots, \alpha_{m}\right) \in \mathbb{R}_{+}^{m}$. Following [HaKn] the polygon space $\operatorname{Pol}(\alpha)$, abelian polygon space $\operatorname{APol}(\alpha)$ and upper path space $\operatorname{UP}(\alpha)$ for $\alpha$ 
are given by

$$
\begin{gathered}
\operatorname{Pol}(\alpha)=\left\{\left(\rho_{1}, \ldots, \rho_{m}\right) \in\left(\mathbb{R}^{3}\right)^{m}|\forall i,| \rho_{i} \mid=\alpha_{i}, \sum_{i=1}^{m} \rho_{i}=0\right\} / S O_{3}, \\
\operatorname{APol}(\alpha)=\left\{\left(\rho_{1}, \ldots, \rho_{m}\right) \in\left(\mathbb{R}^{3}\right)^{m}|\forall i,| \rho_{i} \mid=\alpha_{i}, \zeta\left(\sum_{i=1}^{m} \rho_{i}\right)=\alpha_{m}\right\} / S O_{2}, \\
\operatorname{UP}(\alpha)=\left\{\left(\rho_{1}, \ldots, \rho_{m-1}\right) \in\left(\mathbb{R}^{3}\right)^{m-1}|\forall i,| \rho_{i} \mid=\alpha_{i}, \zeta\left(\sum_{i=1}^{m-1} \rho_{i}\right) \geq \alpha_{m}\right\} / \sim
\end{gathered}
$$

respectively, where $S_{3}$ acts on $\left(\mathbb{R}^{3}\right)^{m}$ diagonally, $\zeta: \mathbb{R}^{3} \rightarrow \mathbb{R}$ is the projection $\zeta(x, y, z)=z$, and $\rho \sim \rho^{\prime}$ if $\rho=\rho^{\prime}$ or if $\zeta\left(\sum_{i=1}^{m-1} \rho_{i}\right)=\alpha_{m}$ and $[\rho]=\left[\rho^{\prime}\right]$ in $\operatorname{APol}(\alpha)$. When $\alpha$ is generic, i.e., the equation $\sum_{i=1}^{m} \varepsilon_{i} \alpha_{i}=0$ has no solution with $\varepsilon_{i}= \pm 1$ they are respectively closed symplectic manifolds of dimensions $2(m-3), 2(m-2)$ and $2(m-1)$. Moreover $\operatorname{Pol}(\alpha)$ is a codimension 2 symplectic submanifold of $\operatorname{APol}(\alpha)$, and the latter is a codimension 2 symplectic submanifold of $\operatorname{UP}(\alpha)$. In particular $\operatorname{APol}(\alpha)$ and $\operatorname{UP}(\alpha)$ are respectively toric manifolds with moment polytopes

$$
\begin{aligned}
& \Xi_{\alpha}=\left\{\left(x_{1}, \ldots, x_{m-1}\right) \in \prod_{i=1}^{m-1}\left[-\alpha_{i}, \alpha_{i}\right] \mid \sum_{i=1}^{m-1} x_{i}=\alpha_{m}\right\}, \\
& \widehat{\Xi}_{\alpha}=\left\{\left(x_{1}, \ldots, x_{m-1}\right) \in \prod_{i=1}^{m-1}\left[-\alpha_{i}, \alpha_{i}\right] \mid \sum_{i=1}^{m-1} x_{i} \geq \alpha_{m}\right\} .
\end{aligned}
$$

Note that $\widehat{\Xi}_{\alpha}$ may viewed as a Delzant polytope. Indeed, with the standard basis $e_{1}, \ldots, e_{m-1}$ of $\mathbb{R}^{m-1}$ we set $u_{i}=e_{i}, u_{m-1+i}=-e_{i}, i=1, \ldots, m-1$ and $u_{2 m-1}=\sum_{i=1}^{m-1} e_{i}$. Then with $\lambda_{m-1+i}=\lambda_{i}=-\alpha_{i}, i=1, \ldots, m-1$, and $\lambda_{2 m-1}=\alpha_{m}$ we have

$$
\widehat{\Xi}_{\alpha}=\bigcap_{k=1}^{2 m-1}\left\{x \in \mathbb{R}^{m-1} \mid\left(x, u_{k}\right)-\lambda_{k} \geq 0\right\} .
$$

For nonnegative integers $\mu_{k}, k=1, \ldots, 2 m-1$ the direct computation gives rise to

$$
\left\{\begin{array}{l}
\sum_{k=1}^{2 m-1} \mu_{k} u_{k}=\sum_{i=1}^{m-1}\left(\mu_{i}-\mu_{m-1+i}+\mu_{2 m-1}\right) e_{i} \\
-\sum_{k=1}^{2 m-1} \lambda_{k} \mu_{k}=\sum_{i=1}^{m-1} \alpha_{i}\left(\mu_{i}+\mu_{m-1+i}\right)-\alpha_{m} \mu_{2 m-1}
\end{array}\right.
$$


So $\sum_{k=1}^{2 m-1} \mu_{k} u_{k}=0 \Leftrightarrow \mu_{m-1+i}=\mu_{i}+\mu_{2 m-1}, i=1, \ldots, m-1$, and thus $1 \leq \sum_{i=1}^{2 m-1} \mu_{i} \leq m \Leftrightarrow 1 \leq 2 \sum_{i=1}^{m-1} \mu_{i}+m \mu_{2 m-1} \leq m$. In this case

$$
-\sum_{k=1}^{2 m-1} \lambda_{k} \mu_{k}=2 \sum_{i=1}^{m-1} \alpha_{i} \mu_{i}+\left(\sum_{i=1}^{m-1} \alpha_{i}-\alpha_{m}\right) \mu_{2 m-1} \text {. }
$$

Setting $b_{i}=\mu_{i}, i=1, \ldots, m-1$, and $b_{m}=\mu_{2 m-1}$ we get

$$
\begin{aligned}
\Lambda\left(\widehat{\Xi}_{\alpha}\right)=2 \pi \max \left\{2 \sum_{i=1}^{m-1} \alpha_{i} b_{i}\right. & +\left(\sum_{i=1}^{m-1} \alpha_{i}-\alpha_{m}\right) b_{m} \mid \\
1 & \left.\leq 2 \sum_{i=1}^{m-1} b_{i}+m b_{m} \leq m, b_{i} \in \mathbb{Z}_{\geq 0}\right\} .
\end{aligned}
$$

By (1.7) it is less than or equal to $2 m \pi \max \left\{\alpha_{i} \mid 1 \leq i \leq m-1\right\}$. Moreover, it is easily checked that $\operatorname{conv}\left(0, \delta e_{1}, \ldots, \delta e_{m-1}\right) \subset \widehat{\Xi}_{\alpha}$ for $\delta=\min \left\{\alpha_{i} \mid 1 \leq\right.$ $i \leq m-1\}$. By Theorem 1.1 we have:

Proposition 5.1. Let $\widehat{\omega}_{\alpha}$ denote the symplectic form on $\operatorname{UP}(\alpha)$. Then for $C=C_{H Z}^{(2)}, C_{H Z}^{(2 \circ)}$,

$$
\begin{aligned}
2 \pi \min \left\{\alpha_{i} \mid 1 \leq i \leq m-1\right\} & \leq \mathcal{W}_{G}\left(\mathrm{UP}(\alpha), \widehat{\omega}_{\alpha}\right) \\
& \leq C\left(\operatorname{UP}(\alpha), \widehat{\omega}_{\alpha} ; p t, P D\left(\left[\widehat{\omega}_{\alpha}\right]\right)\right),
\end{aligned}
$$

and for $m \geq 3$ it holds that

$$
\begin{aligned}
C\left(\mathrm{UP}(\alpha), \widehat{\omega}_{\alpha} ; p t, P D\left(\left[\widehat{\omega}_{\alpha}\right]\right)\right) & \leq \Lambda\left(\widehat{\Xi}_{\alpha}\right) \\
& \leq 2 m \pi \max \left\{\alpha_{i} \mid 1 \leq i \leq m-1\right\} .
\end{aligned}
$$

Since $\Xi_{\alpha}$ is isomorphic to the Delzant polytope

$$
\triangle_{\alpha}=\left\{\left(y_{1}, \ldots, y_{m-2}\right) \in \prod_{i=1}^{m-2}\left[-\alpha_{i}, \alpha_{i}\right] \mid \alpha_{m}-\alpha_{m-1} \leq \sum_{i=1}^{m-2} y_{i} \leq \alpha_{m}+\alpha_{m-1}\right\}
$$

as in the proof of Proposition 5.1 we can derive from Theorem 1.1:

Proposition 5.2. Let $\omega_{\alpha}$ denote the symplectic form on $\operatorname{APol}(\alpha)$. Then

$$
\begin{aligned}
\Lambda\left(\triangle_{\alpha}\right)=\max \left\{2 \sum_{i=1}^{m-2} \mu_{i} \alpha_{i}+\mu_{m-1} \cdot(\right. & \left.\sum_{i=1}^{m-1} \alpha_{i}-\alpha_{m}\right) \\
& \left.+\mu_{m} \cdot\left(\alpha_{m-1}+\alpha_{m}-\sum_{i=1}^{m-2} \alpha_{i}\right)\right\}
\end{aligned}
$$


where $\mu_{i} \in \mathbb{Z}_{\geq 0}, i=1, \ldots, m$, satisfy

$$
1 \leq 2 \sum_{i=1}^{m-2} \mu_{i}+(m-1) \mu_{m-1}+(m-3) \mu_{m} \leq m-1 .
$$

Moreover, for $m \geq 4$ and $C=C_{H Z}^{(2)}, C_{H Z}^{(2 \circ)}$ it holds that

$$
\begin{aligned}
\mathcal{W}_{G}\left(\operatorname{APol}(\alpha), \omega_{\alpha}\right) & \leq C\left(\operatorname{APol}(\alpha), \omega_{\alpha} ; p t, P D\left(\left[\omega_{\alpha}\right]\right)\right) \leq \Lambda\left(\triangle_{\alpha}\right) \\
& \leq 2(m-1) \pi \max \left\{\alpha_{1}, \ldots, \alpha_{m-2}, \alpha_{m-1}+\alpha_{m}\right\}
\end{aligned}
$$

By Proposition 1.3 in [HaKn], for generic $\alpha \in \mathbb{R}_{+}^{m}$ and $\delta>\sum_{j=1}^{m-1} \alpha_{j}$ one has a symplectomorphism

$$
\operatorname{APol}\left(\alpha_{1}, \ldots, \alpha_{m}\right) \cong \operatorname{Pol}\left(\alpha_{1}, \ldots, \alpha_{m-1}, \delta+\alpha_{m}, \delta\right) .
$$

So it follows from Proposition 5.2 that

Proposition 5.3. Let $\omega_{\alpha}^{\prime}$ denote the symplectic form on $\operatorname{Pol}(\alpha)$. Then for every generic $\alpha \in \mathbb{R}_{+}^{m}$ satisfying $\alpha_{m-1}>\alpha_{m}>\frac{1}{2} \sum_{j=1}^{m-1} \alpha_{j}$ the polygon space $\operatorname{Pol}\left(\alpha_{1}, \ldots, \alpha_{m}\right)$ is symplectomorphic to $\operatorname{APol}\left(\alpha_{1}, \ldots, \alpha_{m-2}, \alpha_{m-1}-\right.$ $\left.\alpha_{m}\right)$. So with $\alpha^{\prime}=\left(\alpha_{1}, \ldots, \alpha_{m-1}, \alpha_{m-1}-\alpha_{m}\right)$,

$$
\begin{aligned}
\Lambda\left(\triangle_{\alpha^{\prime}}\right)=\max \left\{2 \sum_{i=1}^{m-3} \mu_{i} \alpha_{i}+\mu_{m-2}\right. & \cdot\left(\sum_{i=1}^{m} \alpha_{i}-2 \alpha_{m-1}\right) \\
& \left.+\mu_{m-1} \cdot\left(2 \alpha_{m-1}+2 \alpha_{m-2}-\sum_{i=1}^{m} \alpha_{i}\right)\right\}
\end{aligned}
$$

where $\mu_{i} \in \mathbb{Z}_{\geq 0}, i=1, \ldots, m-1$, satisfy

$$
1 \leq 2 \sum_{i=1}^{m-3} \mu_{i}+(m-2) \mu_{m-2}+(m-4) \mu_{m-1} \leq m-2
$$

Moreover, for $m \geq 5$ and $C=C_{H Z}^{(2)}, C_{H Z}^{(2 \circ)}$ it holds that

$$
\begin{gathered}
\mathcal{W}_{G}\left(\operatorname{Pol}(\alpha), \omega_{\alpha}^{\prime}\right) \leq C\left(\operatorname{Pol}(\alpha), \omega_{\alpha}^{\prime} ; p t, P D\left(\left[\omega_{\alpha}^{\prime}\right]\right)\right) \leq \Lambda\left(\triangle_{\alpha^{\prime}}\right) \\
\quad \leq 2(m-2) \pi \max \left\{\alpha_{1}, \ldots, \alpha_{m-3}, \alpha_{m-2}+\alpha_{m-1}-\alpha_{m}\right\} .
\end{gathered}
$$

It was shown in Section 6 of [HaKn] that for generic $\alpha \in \mathbb{R}_{+}^{5}$ and $\beta \in \mathbb{R}_{+}^{6}$ both $\operatorname{Pol}(\alpha)$ and $\operatorname{Pol}(\beta)$ are toric manifolds if $\alpha_{1} \neq \alpha_{2}$ and $\alpha_{4} \neq \alpha_{5}$, and if $\beta_{1} \neq \beta_{2}$ and $\beta_{5} \neq \beta_{6}$. 


\section{$\S 6 . \quad$ Related results}

\subsection{Impacts on symplectic capacities of symplectic blow-ups}

If we always require the class $\kappa$ to be a single point one $p t$ in the definition of the number $\operatorname{GW}_{g}\left(M, \omega ; \alpha_{0}, \alpha_{\infty}\right)$ at the beginning of Section 3 , the corresponding infimum is denoted by

$$
\widehat{\mathrm{GW}}_{g}\left(M, \omega ; \alpha_{0}, \alpha_{\infty}\right) \text {. }
$$

Then $\mathrm{GW}_{g}\left(M, \omega ; \alpha_{0}, \alpha_{\infty}\right) \leq \widehat{\mathrm{GW}}_{g}\left(M, \omega ; \alpha_{0}, \alpha_{\infty}\right)$.

Firstly, it is easy to see that the symplectic blow up operation for a symplectic manifold must decrease the volume of it. One easily find a noncompact symplectic manifold for which a suitable symplectic blowing up does not decrease its Gromov symplectic width. Therefore it is a complicated problem. For simplicity we restrict our attention to the case of a symplectic blow up of a closed $2 n$-dimensional symplectic manifold $(M, \omega)$ at $k$ distinct points. Let $\psi=\coprod_{i} \psi_{i}: \coprod_{i}\left(B^{2 n}\left(r_{i}\right), \omega_{0}\right) \rightarrow(M, \omega)$ be a symplectic embedding of $k$ disjoint standard symplectic balls of radii $r_{1}, \ldots, r_{k}$, and $\Theta:\left(\widetilde{M}_{\psi}, \widetilde{\omega}_{\psi}\right) \rightarrow(M, \omega)$ be the symplectic blow-up associated with $\psi$ at $p_{i}=\psi_{i}(0), i=1, \ldots, k$. Let $H_{j}(M)\left(\right.$ resp. $\left.H^{j}(M)\right)$ denote $H_{j}(M, \mathbb{Z})$ $\left(\right.$ resp. $\left.H^{j}(M, \mathbb{Z})\right)$ modulo torsion. Denote by $\Sigma_{i}=\Theta^{-1}(0) \approx \mathbb{C} P^{n-1}$ the exceptional divisor corresponding to $p_{i}$. Let $E_{1}, \ldots, E_{k}$ denote the homology classes of the exceptional divisors in $H_{2 n-2}(\widetilde{M})$ and $e_{1}, \ldots, e_{k} \in H^{2}(\widetilde{M})$ be their Poincaré duals. Then

$$
[\widetilde{\omega}]=\left[\Theta^{*} \omega\right]-\sum_{i=1}^{k} \pi r_{i}^{2} e_{i}
$$

in $H^{2}(\widetilde{M}, \mathbb{R})([\mathrm{McP}])$. Let $E_{i}^{\prime} \in H_{2}(\widetilde{M})$ be the classes of lines in the exceptional divisors $\Sigma_{i}$ such that $P D\left(E_{i}^{\prime}\right)=-\left(-e_{i}\right)^{n-1}$. Let $\left\{T_{0}, \ldots, T_{q}\right\}$ be a homogeneous basis of $H^{2}(M)$ of increasing codimension such that $T_{0}$ is the fundamental class and $T_{q}=p t$. With $p=q+k(n-1)$ we define $\tilde{T}_{q+1}, \ldots, \tilde{T}_{p}$ to be the classes $e_{i}^{j} \in H^{2}(\widetilde{M}), i=1, \ldots, k$ and $j=1, \ldots, n-1$. Denote by $\tilde{T}_{i}=\Theta^{*} T_{i}, i=1, \ldots, q$. Then $\left\{\tilde{T}_{1}, \ldots, \tilde{T}_{p}\right\}$ is a homogeneous basis of $H^{2}(\widetilde{M})$. The classes $\tilde{T}_{1}, \ldots, \tilde{T}_{q}$ (resp. $\left.\tilde{T}_{q+1}, \ldots, \tilde{T}_{p}\right)$ are called nonexceptional (resp. exceptional). Note that

$$
\begin{aligned}
& \tilde{T}_{j} \cdot \tilde{T}_{j^{\prime}}=\Theta^{*}\left(T_{j} \cdot T_{j^{\prime}}\right), \quad \tilde{T}_{j} \cdot e_{i}^{m}=0 \\
& e_{i}^{m} \cdot e_{i^{\prime}}^{m^{\prime}}=\delta_{i^{\prime}}^{i} e_{i}^{m+m^{\prime}}, \quad e_{i}^{n}=(-1)^{n-1} p t
\end{aligned}
$$


on $\widetilde{M}$ for $1 \leq j, j^{\prime} \leq q, 1 \leq i, i^{\prime} \leq k$ and $1 \leq m, m^{\prime} \leq n-1$. One has a canonical decomposition

$$
H_{2}(\widetilde{M})=H_{2}(M) \oplus \mathbb{Z} E_{1}^{\prime} \oplus \cdots \oplus \mathbb{Z} E_{k}^{\prime}
$$

By (6.3) the classes $P D\left(E_{i}^{\prime}\right) \in H^{2}(\widetilde{M})$ satisfy $P D\left(E_{i}^{\prime}\right) \cdot E_{j}=E_{j}\left(E_{i}^{\prime}\right)=-\delta_{j}^{i}$. So one has

$$
H^{2}(\widetilde{M}, \mathbb{R})=H^{2}(M, \mathbb{R}) \oplus \mathbb{R} e_{1}^{n-1} \oplus \cdots \oplus \mathbb{R} e_{k}^{n-1}
$$

As usual we denote by $\Theta$ ! the transfer map $P D_{\widetilde{M}} \circ \Theta^{*} \circ P D_{M}$ from $H_{*}(M)$ to $H_{*}(\widetilde{M})$ and call the image $\Theta !(A)$ the corresponding non-exceptional class of $A \in H_{2}(M)$. Using (6.2) and (6.3) it is not hard to derive that

$$
\langle[\widetilde{\omega}], \Theta !(A)\rangle=\langle[\omega], A\rangle .
$$

Let $p t_{M}\left(\right.$ resp. $\left.p t_{\widetilde{M}}\right)$ denote the single point class in $H_{0}(M)\left(\operatorname{resp} . H_{0}(\widetilde{M})\right)$ such that $\left\langle P D_{M}\left(p t_{M}\right),[M]\right\rangle=1\left(\operatorname{resp} .\left\langle P D_{\widetilde{M}}\left(p t_{\widetilde{M}}\right),[\widetilde{M}]\right\rangle=1\right)$. Note that $H^{2 n}(\widetilde{M}, \mathbb{R})=\mathbb{R}\left[\widetilde{\omega}^{n}\right]$ and $H^{2 n}(M, \mathbb{R})=\mathbb{R}\left[\omega^{n}\right]$. It is easily checked that

$$
\Theta !\left(p t_{M}\right)=p t_{\widetilde{M}} \text { and } \sum_{i=1}^{k} r_{i}^{2 n}\left(-\pi e_{i}\right)^{n}=-\left(\pi^{n} \sum_{i=1}^{k} r_{i}^{2 n}\right) P D_{\widetilde{M}}\left(p t_{\widetilde{M}}\right)
$$

It was proved in $[\mathrm{Ga}]$ and $[\mathrm{Hu}]$ that

$$
\Psi_{\Theta !(A), 0, m}^{\widetilde{M}}\left(p t ; \Theta !\left(\gamma_{1}\right), \ldots, \Theta !\left(\gamma_{m}\right)\right)=\Psi_{A, 0, m}^{M}\left(p t ; \gamma_{1}, \ldots, \gamma_{m}\right)
$$

for any $A \in H_{2}(M)$ and $\gamma_{i} \in H_{*}(\widetilde{M}), i=1, \ldots, m$. We here use the homology classes for convenience. It follows from this, (6.1) and (6.4) that

$$
\widehat{\mathrm{GW}}_{0}\left(\widetilde{M}, \widetilde{\omega} ; \Theta !\left(\alpha_{0}\right), \Theta !\left(\alpha_{\infty}\right)\right) \leq \widehat{\mathrm{GW}}_{0}\left(M, \omega ; \alpha_{0}, \alpha_{\infty}\right)
$$

Note that the first identity in (6.5) and (6.6) give

$$
\begin{aligned}
& \Psi_{\Theta !(A), 0, m+1}^{\widetilde{M}}\left(p t ; p t_{\widetilde{M}}, \Theta !\left(\gamma_{1}\right), \ldots, \Theta !\left(\gamma_{m}\right)\right) \\
& \quad=\Psi_{A, 0, m+1}^{M}\left(p t ; p t_{M}, \gamma_{1}, \ldots, \gamma_{m}\right) .
\end{aligned}
$$

By $(3.2), \mathrm{GW}_{0}\left(\widetilde{M}, \widetilde{\omega} ; p t_{\widetilde{M}}, P D([\widetilde{\omega}])\right)$ is equal to the infimum of the $\widetilde{\omega}$-areas $\widetilde{\omega}(A)$ of the classes $A \in H_{2}(\widetilde{M})$ for which $\Psi_{A, 0, m+1}^{\widetilde{M}}\left(\kappa ; p t_{\widetilde{M}}, \beta_{1}, \ldots, \beta_{m}\right) \neq 0$ 
for some classes $\beta_{1}, \ldots, \beta_{m} \in H_{*}(\widetilde{M} ; \mathbb{Q}), \kappa \in H_{*}\left(\overline{\mathcal{M}}_{0, m+1} ; \mathbb{Q}\right)$ and integer $m>1$. Hence it follows from (6.8) that

$$
\begin{aligned}
& \mathrm{GW}_{0}\left(\widetilde{M}, \widetilde{\omega} ; p t_{\widetilde{M}}, P D([\widetilde{\omega}])\right) \\
& \quad \leq \inf \left\{\omega(A) \mid \Psi_{A, 0, m+1}^{M}\left(p t ; p t_{M}, \gamma_{1}, \ldots, \gamma_{m}\right) \neq 0\right\}
\end{aligned}
$$

where the infimum is taken for $A \in H_{2}(M)$ and $\gamma_{i} \in H_{*}(M)$. By (3.1) and (6.7) we obtain:

TheOREM 6.1. For any nonzero classes $\alpha_{0}, \alpha_{\infty} \in H_{*}(M, \mathbb{Q})$ it holds that

$$
\begin{aligned}
& C_{H Z}\left(\widetilde{M}, \widetilde{\omega} ; \Theta !\left(\alpha_{0}\right), \Theta !\left(\alpha_{\infty}\right)\right) \leq \widehat{\mathrm{GW}}_{0}\left(M, \omega ; \alpha_{0}, \alpha_{\infty}\right) \quad \text { and } \\
& C_{H Z}\left(\widetilde{M}, \widetilde{\omega} ; p t_{\widetilde{M}}, P D([\widetilde{\omega}])\right) \\
& \quad \leq \inf \left\{\omega(A) \mid \Psi_{A, 0, m+1}^{M}\left(p t ; p t_{M}, \gamma_{1}, \ldots, \gamma_{m}\right) \neq 0\right\} .
\end{aligned}
$$

Notice that the blow-ups of a toric manifold at its toric fixed points are also toric manifolds. However, the blow up of a toric Fano manifold is not necessarily Fano again. By (3.7) and Theorem 6.1 we get:

THEOREM 6.2. Let $X_{\widetilde{\Sigma}}$ be a toric manifold obtained by a sequence of blowings up of a toric Fano manifold at toric fixed points. So $G(\Sigma)=$ $\left\{u_{1}, \ldots, u_{d}\right\} \subset G(\widetilde{\Sigma})$. Then for any strictly convex support function $\varphi$ for $\widetilde{\Sigma}$ (also strictly convex for $\Sigma$ ) it holds that

$$
\mathcal{W}_{G}\left(X_{\widetilde{\Sigma}}, \varphi\right) \leq C\left(X_{\widetilde{\Sigma}}, \varphi ; p t, P D([\varphi])\right) \leq 2 \pi \cdot \Upsilon(\Sigma, \varphi)
$$

for every $n \geq 2$ and $C=C_{H Z}^{(2)}, C_{H Z}^{(2 \circ)}$. Here $\Upsilon(\Sigma, \varphi)$ is given by (1.10) and is always more than zero though $\Upsilon(\widetilde{\Sigma}, \varphi)$ might equal to zero in the case $X_{\widetilde{\Sigma}}$ is not Fano.

The fan $\widetilde{\Sigma}$ may be obtained from $\Sigma$ by a sequence of regular stellar operations. For the Delzant polytope $\triangle$ in (1.3) and a vertex $p$ of it, let the rays $p+t v_{i}, t \geq 0$, form the edges of $\triangle$ at $p$ as in the definition of Delzant polytope above, we choose $0<\varepsilon<E_{p}(\triangle)$ and replace the vertex $p$ by the $n$ vertices $p+\varepsilon v_{i}, i=1, \ldots, n$ to get a new Delzant polytope $\triangle_{\varepsilon}$. Then the symplectic toric manifold $\left(M_{\triangle_{\varepsilon}}, \omega_{\triangle_{\varepsilon}}\right)$ is the symplectic $\varepsilon$ blow-up of the symplectic toric manifold $\left(M_{\triangle}, \omega_{\triangle}, \tau_{\triangle}, \mu_{\triangle}\right)$ at a fixed point $q=\mu_{\triangle}(p)$ of the $\mathbb{T}^{n}$-action $\tau_{\triangle}$. 
Corollary 6.3. Suppose that there exist $r>0$ and $m \in\left(\mathbb{R}^{n}\right)^{*}$ such that $r \cdot(m+\triangle)$ satisfies (1.15), i.e., $M_{\triangle}$ is Fano. Then for $C=C_{H Z}^{(2)}$, $C_{H Z}^{(2 \circ)}$ and any $n \geq 2$ it holds that

$$
\mathcal{W}_{G}\left(M_{\triangle_{\varepsilon}}, \omega_{\triangle_{\varepsilon}}\right) \leq C\left(M_{\triangle_{\varepsilon}}, \omega_{\triangle_{\varepsilon}} ; p t, P D\left(\left[\omega_{\triangle_{\varepsilon}}\right]\right)\right) \leq 2 \pi \Upsilon(\triangle)
$$

\subsection{Symplectic packings in symplectic toric manifolds}

We here presents a symplectic packing result in symplectic toric manifolds via symplectic ellipsoid of form (3.8) (see [Bi], [Gr], [McP], [Ka], [Tr], [Sch] and references therein for the exposition and related results). Denote by $\operatorname{Vert}(\triangle)$ the vertex set of the Delzant polytope $\triangle$ in (1.3). For each $p \in \operatorname{Vert}(\triangle)$ let $p_{1}, \ldots, p_{n}$ be its adjacent $n$ vertexes. If $\sharp \operatorname{Vert}(\triangle)>n+1$ there must exist another $p^{\prime} \in \operatorname{Vert}(\triangle)$ and adjacent $n$ vertexes $p_{1}^{\prime}, \ldots, p_{n}^{\prime}$ corresponding to it such that

$$
\left(\operatorname{conv}\left(p, p_{1}, \ldots, p_{n}\right)\right)^{\circ} \cap\left(\operatorname{conv}\left(p^{\prime}, p_{1}^{\prime}, \ldots, p_{n}^{\prime}\right)\right)^{\circ}=\emptyset .
$$

Hereafter $S^{\circ}$ denotes the interior of the set $S$. In this case we say that the vertexes $q$ and $q^{\prime}$ are simplicially separating in $\triangle$. Notice also that each $\operatorname{conv}\left(p, p_{1}, \ldots, p_{n}\right)$ determines a family of open symplectic ellipsoids $E(\triangle, p, \epsilon):=E\left(\sqrt{2 r_{p}(\triangle)_{1}}-\epsilon, \ldots, \sqrt{2 r_{p}(\triangle)_{n}}-\epsilon\right)$ for $0 \leq \epsilon \leq E_{p}(\triangle)$.

TheOREM 6.4. If any two points of a given subset $\left\{q_{1}, \ldots, q_{m}\right\} \subset$ $\operatorname{Vert}(\triangle)$ are simplicially separating in $\triangle$, then for any small $\epsilon>0$ there exists a symplectic packing of $\left(M_{\triangle}, \omega_{\triangle}\right)$ via the ellipsoids $E\left(\triangle, q_{k}, \epsilon\right), k=$ $1, \ldots, m$.

Proof. By (2.3) it suffices to prove that for any small $\epsilon>0$ there exists a symplectic embedding of a disjoint union of the open ellipsoids $E\left(\triangle, q_{k}, \epsilon\right)$, $k=1, \ldots, m$. For each $k=1, \ldots, m$, as in proof of Proposition 1.3 we have the unimodular matrixes $A_{k} \in \mathrm{SL}(n, \mathbb{Z})$ such that the corresponding transformations

$$
\Phi_{k}:\left(\mathbb{R}^{n}\right)^{*} \longrightarrow\left(\mathbb{R}^{n}\right)^{*}, \quad x \longmapsto A_{k} x-q_{k},
$$

$\operatorname{map} q_{k}, p_{k 1}, \ldots, p_{k n}$ to $0, a_{k 1} e_{1}^{*}, \ldots, a_{k n} e_{n}^{*}, k=1, \ldots, m$, respectively. Here $p_{k 1}, \ldots, p_{k n}$ are the adjacent $n$ vertexes to $q_{k}$, and $a_{k i}=r_{q_{k}}(\triangle)_{i}, i=$ $1, \ldots, n$, and $k=1, \ldots, m$. Now each $\Phi_{k}$ induces a symplectomorphism $\mathcal{A}_{k}$ of $\left(\left(\mathbb{R}^{n}\right)^{*} \times \mathbb{T}^{n}, \omega_{\text {can }}\right)$ to itself that maps $\operatorname{conv}\left(q_{k}, p_{k 1}, \ldots, p_{k n}\right) \times \mathbb{T}^{n}$ onto $\operatorname{conv}\left(0, a_{k 1} e_{1}^{*}, \ldots, a_{k n} e_{n}^{*}\right) \times \mathbb{T}^{n}, k=1, \ldots, m$. Note that

$$
\left(\operatorname{conv}\left(0, a_{k 1} e_{1}^{*}, \ldots, a_{k n} e_{n}^{*}\right)\right)^{\circ}=\triangle\left(a_{k 1}, \ldots, a_{k n}\right), \quad k=1, \ldots, m,
$$


provided that $\left(\mathbb{R}^{n}\right)^{*}$ is identified with $\mathbb{R}^{n}$ by the isomorphism

$$
x_{1} e_{1}^{*}+\cdots+x_{n} e_{n}^{*} \longmapsto x_{1} e_{1}+\cdots+x_{n} e_{n} .
$$

We can use Lemma 3.5 to find symplectic embeddings $\mathcal{B}_{k}$ of

$$
E\left(\triangle, q_{k}, \epsilon\right)=E\left(\sqrt{2 a_{k 1}}-\epsilon, \ldots, \sqrt{2 a_{k n}}-\epsilon\right)
$$

into $\left(\triangle\left(a_{k 1}, \ldots, a_{k n}\right) \times \square^{n}(2 \pi), \omega_{0}\right)$ and thus into $\left(\triangle\left(a_{k 1}, \ldots, a_{k n}\right) \times \mathbb{T}^{n}\right.$, $\left.\omega_{\text {can }}\right), k=1, \ldots, m$. Then it is easily checked that the compositions $\mathcal{A}_{k}^{-1}$ 。 $\mathcal{B}_{k}, k=1, \ldots, m$, give the desired symplectic embeddings.

Remark 6.5. Let $\underline{a}=\left(a_{1}, \ldots, a_{n}\right)$ be a vector of positive weights and $\triangle^{n}(\underline{a}):=\triangle\left(a_{1}, \ldots, a_{n}\right)$. Also denote by $E(\sqrt{2 \underline{a}}):=E\left(\sqrt{2 a_{1}}, \ldots, \sqrt{2 a_{n}}\right)$. The above proof actually shows that if for some $\triangle^{n}\left(\underline{a}^{(k)}\right) \subset \mathbb{R}^{n} \equiv\left(\mathbb{R}^{n}\right)^{*}$ there exist $A_{k} \in \mathrm{SL}(n, \mathbb{Z})$ and $q_{k} \in\left(\mathbb{R}^{n}\right)^{*}, k=1, \ldots, m$, such that the sets $A_{k}\left(\triangle^{n}\left(\underline{a}^{(k)}\right)\right)+q_{k} \subset \triangle, k=1, \ldots, m$, are mutually disjoint, then $\left(M_{\triangle}, \omega_{\triangle}\right)$ admits a symplectic packing via $m$ open ellipsoids $E\left(\sqrt{2 \underline{a}^{(k)}}\right), k=1, \ldots, m$.

ExAmple 6.6. Consider the polygon space $\left(\operatorname{Pol}(\alpha), \omega_{\alpha}\right)$ in Remark 1.5. Its moment polytope $\triangle_{\alpha}$ has vertexes: $q_{1}=(1 / 2,3 / 2), q_{2}=(4 / 3,7 / 3)$, $q_{3}=(5 / 2,7 / 3), q_{4}=(5 / 2,3 / 2), q_{5}=(4 / 3,1 / 3), q_{6}=(2 / 3,1 / 3)$ and $q_{7}=$ $(1 / 2,1 / 2)$. It is easily computed that

$$
\begin{array}{ll}
E\left(\triangle, q_{1}\right)=E(\sqrt{2}, \sqrt{5 \sqrt{2} / 3}), & E\left(\triangle, q_{2}\right)=E(\sqrt{5 \sqrt{2} / 3}, \sqrt{7 / 3}), \\
E\left(\triangle, q_{3}\right)=E(\sqrt{7 / 3}, \sqrt{5 / 3}), & E\left(\triangle, q_{4}\right)=E(\sqrt{5 / 3}, \sqrt{7 \sqrt{2} / 3}), \\
E\left(\triangle, q_{5}\right)=E(\sqrt{7 \sqrt{2} / 3}, \sqrt{4 / 3}), & E\left(\triangle, q_{6}\right)=E(\sqrt{4 / 3}, \sqrt{\sqrt{10} / 3}), \\
E\left(\triangle, q_{7}\right)=E(\sqrt{\sqrt{10} / 3}, \sqrt{2}) . &
\end{array}
$$

By Theorem 6.4, for any $\epsilon>0$ sufficiently small, $\left(\operatorname{Pol}(\alpha), \omega_{\alpha}\right)$ admits the symplectic packings via the following groups of ellipsoids:

$$
\begin{aligned}
& \left\{E\left(\triangle, q_{1}, \epsilon\right), E\left(\triangle, q_{3}, \epsilon\right), E\left(\triangle, q_{5}, \epsilon\right)\right\},\left\{E\left(\triangle, q_{1}, \epsilon\right), E\left(\triangle, q_{3}, \epsilon\right), E\left(\triangle, q_{6}, \epsilon\right)\right\}, \\
& \left\{E\left(\triangle, q_{2}, \epsilon\right), E\left(\triangle, q_{4}, \epsilon\right), E\left(\triangle, q_{6}, \epsilon\right)\right\},\left\{E\left(\triangle, q_{2}, \epsilon\right), E\left(\triangle, q_{4}, \epsilon\right), E\left(\triangle, q_{7}, \epsilon\right)\right\}, \\
& \left\{E\left(\triangle, q_{3}, \epsilon\right), E\left(\triangle, q_{5}, \epsilon\right), E\left(\triangle, q_{7}, \epsilon\right)\right\},\left\{E\left(\triangle, q_{1}, \epsilon\right), E\left(\triangle, q_{4}, \epsilon\right), E\left(\triangle, q_{6}, \epsilon\right)\right\}, \\
& \left\{E\left(\triangle, q_{2}, \epsilon\right), E\left(\triangle, q_{5}, \epsilon\right), E\left(\triangle, q_{7}, \epsilon\right)\right\} .
\end{aligned}
$$




\subsection{Seshadri constants}

Let $(M, J)$ be a compact complex manifold of dimension $n$, and $L \rightarrow M$ an ample line bundle. Demailly [Dem] defined the Seshadri constant of $L$ at a point $x \in M$ to be the infimum $\varepsilon(L, x)$ of $\int_{C} c_{1}(L) /$ mult $_{x} C$, where $C$ takes over all irreducible curves passing through the point $x$, and $\operatorname{mult}_{x} C$ is the multiplicity of $C$ at $x$. The global Seshadri constant is defined by $\varepsilon(L):=\inf _{x \in M} \varepsilon(L, x)$. For the toric manifold $X_{\Sigma}$ as in Theorem 1.1 let $L_{k} \rightarrow P_{\triangle}$ be the corresponding line bundles to the toric divisors $D_{k}(\Sigma)$ in $(2.5), k=1, \ldots, d$. It is well-known that the Chern class $c_{1}\left(L_{k}\right)$ is Poincaré dual to $\left[D_{k}\right] \in H_{2}\left(X_{\Sigma}, \mathbb{Z}\right)$ for each $k$.

THEOREM 6.7. Let $\Sigma$ be a complete regular fan in $\mathbb{R}^{n}$. Then for any ample line bundle $L \rightarrow X_{\Sigma}$ and any strictly convex support function $\varphi_{L}$ representing the class $c_{1}(L)$ it holds that

$$
\varepsilon(L) \leq 2 \pi \cdot \Lambda\left(\Sigma, \varphi_{L}\right)
$$

Furthermore, if $X_{\Sigma}$ is also Fano then

$$
\varepsilon(L) \leq 2 \pi \cdot \Upsilon\left(\Sigma, \varphi_{L}\right)
$$

Proof. Recall that in Definition 1.26 of [Lu3, v9] we defined

$$
\operatorname{GW}(M, \omega)=\inf \mathrm{GW}_{g}(M, \omega ; p t, \alpha)
$$

where the infimum is taken over all nonnegative integers $g$ and all homology classes $\alpha \in H_{*}(M ; \mathbb{Q}) \backslash\{0\}$ of degree $\operatorname{deg} \alpha \leq \operatorname{dim} M-1$. Using Proposition 6.3 in [BiCi] we showed in Theorem 1.36 of [Lu3, v9] that for a closed connected complex manifold $(M, J)$ of dimension $\operatorname{dim}_{\mathbb{R}} M>2$ and any ample line bundle $L \rightarrow M$ it holds that $\varepsilon(L) \leq \mathrm{GW}\left(M, \omega_{L}\right)$. Here $\omega_{L}$ is any $J$-compatible Kähler form (the curvature form for a suitable metric connection on $L$ ) representing the cohomology class $c_{1}(L)$. From the proofs of Theorems 1.1 and 1.2 it is easily seen that for a toric manifold $X_{\Sigma}$ and a strictly convex support $\varphi$ for $\Sigma$ one has

$$
\mathrm{GW}\left(X_{\Sigma}, \varphi\right) \leq 2 \pi \Lambda(\Sigma, \varphi) \quad \text { and } \quad \mathrm{GW}\left(X_{\Sigma}, \varphi\right) \leq 2 \pi \Upsilon(\Sigma, \varphi)
$$

in general case and Fano case respectively. 


\subsection{Symplectic capacities of symplectic manifolds with $S^{1}$ - action}

The symplectic toric manifolds are a special class of symplectic manifolds with the Hamiltonian $S^{1}$-action. Let $\left\{\lambda_{t}\right\}=\lambda: S^{1}=\mathbb{R} / \mathbb{Z} \rightarrow$ $\operatorname{Ham}(M, \omega)$ be a Hamiltonian circle action on a connected symplectic manifold of dimension $2 n$. Let $H: M \rightarrow \mathbb{R}$ be the Hamiltonian function for the action. It means that the circle action is generated by the Hamiltonian vector field $X_{H}$. This action is called semi-free if it is free on $M \backslash M^{S^{1}}$. For each fixed point $p$ of the action there exist integers $m_{1}, \ldots, m_{n}$ such that the induced linear symplectic $S^{1}$-action on the tangent space $T_{p} M$ is isomorphic to the action on $\left(\mathbb{C}^{n}, \omega_{0}\right)$ generated by the moment map

$$
H_{0}\left(z_{1}, \ldots, z_{n}\right)=\pi \sum_{j=1}^{n} m_{j}\left|z_{j}\right|^{2} .
$$

The integers $m_{1}, \ldots, m_{n}$, uniquely determined up to permutation, are called the isotropy weights at $p$. An Hamiltonian $S^{1}$-action on $(M, \omega)$ is semi-free if and only if the only isotropy weights at every fixed point are \pm 1 .

TheOrem 6.8. Let $(M, \omega)$ be a $2 n$-dimensional, connected closed symplectic manifold with a semi-free Hamiltonian circle action with isolated fixed points. Then

$$
\mathcal{W}_{G}(M, \omega) \leq C(M, \omega ; p t, P D([\omega])) \leq \max H-\min H
$$

for $C=C_{H Z}^{(2)}, C_{H Z}^{(2 \circ)}$ and any $n \geq 2$, where $H$ is the associated Hamiltonian function. Moreover, if $[\omega] \in H^{2}(M, \mathbb{Q})$ and the only isotropy weights at every fixed point is \pm 1 then

$$
\mathcal{W}_{G}(M, \omega) \geq \frac{\pi}{m}
$$

Here $m>0$ is the smallest integer such that $m[\omega] \in H^{2}(M, \mathbb{Z})$.

Proof. Following the notations in $[\mathrm{Go}]$ let $\mathcal{S}=\{1, \ldots, n\}$. Each subset $I \subset \mathcal{S}$ may determine a homology class $A_{I} \in H_{2}(M)$ in Proposition 2.11 of [Go] such that $\omega\left(A_{I}\right)=\max H-H\left(p_{I^{c}}\right)$ with $I^{c}=\mathcal{S} \backslash I$. By (14) in Corollary 3.14 of $[\mathrm{Go}]$ one has $x_{\mathcal{S}} * x_{I}=x_{I^{c}} \otimes e^{A_{I}}$. It follows that GromovWitten invariant

$$
\Psi_{A_{I}, 0,3}\left(p t ; P D\left(x_{\mathcal{S}}\right), P D\left(x_{I}\right), P D\left(x_{J}\right)\right) \neq 0
$$


for some $J \subset \mathcal{S}$. Note that $x_{\mathcal{S}}$ is the positive generator $H^{2 n}(M, \mathbb{Z}$ ) (cf. Remark 2.10 in [Go].) (6.16) shows that $(M, \omega)$ is strong 0-symplectic uniruled in the sense of Definition 1.14 in [Lu3, v9]. As in [Lu1] and [Lu3], using the the reduction formula of the Gromov-Witten invariants we can also derive from (6.16) that

$$
\Psi_{A_{I}, 0,4}\left(\pi^{-1}(p t) ; p t, P D([\omega]), \alpha\right) \neq 0
$$

for some $\alpha \in H_{*}(M, \mathbb{R})$. So it follows from (12) and Theorem 1.13 in [Lu3, v9] that

$$
\begin{aligned}
\mathcal{W}_{G}(M, \omega) & \leq C_{H Z}^{(2)}(M, \omega ; p t, P D([\omega])) \\
& \leq C_{H Z}^{(2 \circ)}(M, \omega ; p t, P D([\omega])) \\
& \leq G W_{0}(M, \omega ; p t, P D([\omega])) \\
& \leq \omega\left(A_{I}\right) \\
& \leq \max H-H\left(p_{I^{c}}\right) \\
& \leq \max H-\min H .
\end{aligned}
$$

(6.14) is proved.

For the second claim, by Proposition 2.8 in [KaTo] there exists a symplectic embedding from $\left(B^{2 n}(1), \omega_{0}\right)$ to $(M, m \omega)$. So $\mathcal{W}_{G}(M, m \omega) \geq \pi$. (6.15) follows.

\section{REFERENCES}

[Ab] M. Abreu, Kähler geometry of toric manifolds in symplectic coordinates, Symplectic and contact topology: interactions and perspectives (Toronto, ON/Montreal, QC, 2001), Fields Inst. Commun. 35, Amer. Math. Soc., Providence, RI (2003), pp. 1-24.

[Au] M. Audin, The topology of torus actions on symplectic manifolds, Progress in Mathematics, 93, Birkhäuser, 1991.

[Ba1] V. V. Batyrev, Quantum cohomology rings of toric manifolds, Astérisque, 218 (1993), 9-34.

[Ba2] V. V. Batyrev, On the classification of smooth projective toric varieties, J. Algebraic Geometry, 3 (1994), 493-535.

[Ba3] V. V. Batyrev, On the classification of toric Fano 4-folds, J. Math. Sciences, 94 (1999), 1021-1050.

[Bi] P. Biran, From symplectic packing to algebraic geometry and back, European Congress of Mathematics, Vol. II (Barcelona, 2000), Prog. Math., 202, Birkhäuser (2001), pp. 507-524. 
[BiCi] P. Biran and K. Cieliebak, Symplectic topology on subcritical manifolds, Comm. Math. Helv., 76 (2001), no. 4, 712-753.

[CdFKM] P. Candelas, X. de la Ossa, A. Font, S. Katz and D. Morrison, Mirror symmetry for two parameter models I, Mirror symmetry, II, AMS/IP Stud. Adv. Math., 1, Amer. Math. Soc., Providence, RI (1997), pp. 483-543.

[CiS] K. Cieliebak and D. A. Salamon, Wall crossing for symplectic vortices and quantum cohomology, math.SG/0209170.

[Del] T. Delzant, Hamiltoniens périodiques et image convexe de l'application moment, Bull. Soc. Math. France, 116 (1988), 315-339.

[Dem] J.-P. Demailly, $L^{2}$-vanishing theorems for positive line bundles and adjunction theory, Transcendental methods in Algebraic Geometry (F. Catanese and C. Ciliberto, eds.), Lect. Notes Math. 1646, Springer-Verlag (1992), pp. 1-97.

[Ew] G. Ewald, Combinatorial combinatorial convexity and algebraic geometry, Graduate Texts in Mathematics 168, Springer, 1996.

[Fu] W. Fulton, Introduction to Toric Varieties, Annals of Mathematics Studies 131, Princeton University Press, 1993.

[Ga] A. Gathmann, Gromov-Witten invariants of blow-ups, J. Algebraic Geom., 10 (2001), no. 3, 399-432.

[Gin] V. Ginzburg, The Weinstein conjecture and the theorems of nearby and almost existence, The breadth of symplectic and Poisson geometry, Progr. Math., 232, Birkhäuser Boston, Boston, MA (2005), pp. 139-172.

[Giv] A. Givental, A mirror theorem for toric complete intersections, Topological field theory, primitive forms and related topics (Kyoto, 1996), Progr. Math., 160, Birkhäuser Boston, Boston, MA (1998), pp. 141-175.

[Go] E. Gonzalez, Quantum cohomology and $S^{1}$-action with isolated fixed points, math.SG/0310114.

[Gr] M. Gromov, Pseudoholomorphic curves in symplectic manifolds, Invent. Math., 82 (1985), 307-347.

[Gu1] V. Guillemin, Kähler structures on toric varieties, J. Diff. Geom., 40 (1994), 285-309.

[Gu2] V. Guillemin, Moment maps and combinatorial invariants of Hamiltonian $\mathbb{T}^{n}$-spaces, Progress in Mathematics, 122, Birkhäuser, 1994.

[HaKn] J.-C. Hausmann and A. Knutson, The cohomology ring of polygon spaces, Ann. Inst. Fourier, Grenoble, 48 (1998), 281-321.

[Hu] J. Hu, Gromov-Witten invariants of blow-ups along points and curves, Math. Z., 233 (2000), no. 4, 709-739.

[HZ] H. Hofer and E. Zehnder, Symplectic Invariants and Hamiltonian Dynamics, Birkhäuser, Boston, MA, 1994.

[Ka] Y. Karshon, Appendix to [McP], Inven. Math., 115 (1994), 431-434.

[KaTo] Y. Karshon and S. Tolman, The Gromov width of complex Grassmannians, math.SG/0405391.

[Ko] J. Kollár, Low Degree Polynomial Equations: Arithmetic, Geometry and Topology, Progress in Mathematics, 122, Birkhäuser (1994), pp. 255-288. 
[KoMor] J. Kollár and S. Mori, Birational Geometry of Algebraic Varieties, Cambridge tracts in Mathematics, 134, Cambridge University Press, 1998.

[Kr] A. Kresch, Gromov-Witten invariants of a class of toric varietes, Michigan Math. J., 48 (2000), 369-391.

[Lu1] G. C. Lu, The Weinstein conjecture in the uniruled manifolds, Math. Res. Lett., 7 (2000), 383-387.

[Lu2] G. C. Lu, Symplectic capacities of toric manifolds and combinatorial inequalities, C. R. Acad. Sci. Paris, Ser. I, 334 (2002), 889-892.

[Lu3] G. C. Lu, Gromov-Witten invariants and pseudo symplectic capacities, math.SG/0103195, v6, 6 September 2001, and v9, 3 December 2004, to appear in Israel Journal of Mathematics.

[Mc] D. McDuff, Quantum homology of fibrations over $S^{2}$, International Journal of mathematics, 11 (2000), 665-721.

[McP] D. McDuff and L. Polterovich, Symplectic packings and algebraic geometry, Invent. Math., 115 (1994), 405-425.

[Mor1] S. Mori, Projective manifolds with ample tangent bundles, Ann. Math., 110 (1975), 593-606.

[Mor2] S. Mori, An email communication.

[Oda] T. Oda, Convex Bodies and Algebraic Geometry, Springer-Verlag, 1988.

[Sa] H. Sato, Toward the classification of higher-dimensional toric Fano varieties, Tohoku Math. J., 52 (2000), no. 3, 383-413.

[Sch] F. Schlenk, On symplectic folding, preprint, math.SG/9903086, March 1999.

[Sik] J. C. Sikorav, Rigidité symplectique dans le cotangent de $\mathbb{T}^{n}$, Duke Mathematical Journal, 59 (1989), 227-231.

[Sp] H. Spielberg, The Gromov-Witten invariants of symplectic toric manifolds, and their quantum cohomology ring, C. R. Acad. Sci. Paris, Ser. I, 329 (1999), 699-704.

[Tr] L. Traynor, Symplectic packing constructions, J. Diff. Geom., 41 (1995), 735-751.

[Wi] J. A. Wiśniewski, Toric Mori theory and Fano manifolds, Séminaires \& Congrès, 6 (2002), 249-272.

Department of Mathematics

Beijing Normal University

Beijing 100875

P. R. China

gclu@bnu.edu.cn 